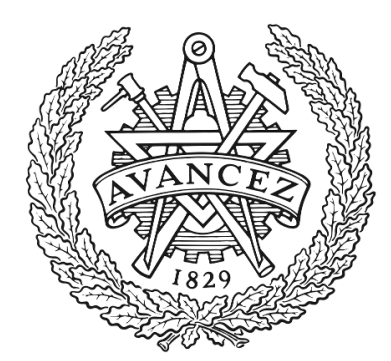

\title{
CHALMERS
}

UNIVERSITY OF TECHNOLOGY

\section{Distributed Direct Localization Suitable for Dense Networks}

Downloaded from: https://research.chalmers.se, 2023-04-26 09:52 UTC

Citation for the original published paper (version of record):

Zhang, S., Staudinger, E., Jost, T. et al (2020). Distributed Direct Localization Suitable for Dense Networks. IEEE Transactions on Aerospace and Electronic Systems, 56(2): 1209-1227.

http://dx.doi.org/10.1109/TAES.2019.2928606

N.B. When citing this work, cite the original published paper.

(C2020 IEEE. Personal use of this material is permitted.

However, permission to reprint/republish this material for advertising or promotional purposes 


\title{
Distributed Direct Localization Suitable for Dense Networks
}

\author{
Siwei Zhang, Member, IEEE, Emanuel Staudinger, Thomas Jost, Member, IEEE, Wei Wang, Member, IEEE, \\ Christian Gentner, Armin Dammann, Member, IEEE, Henk Wymeersch, Member, IEEE, \\ and Peter Adam Hoeher, Fellow, IEEE
}

\begin{abstract}
Traditional network localization algorithms contain ranging and localization steps, which have systematic disadvantages. We propose an algorithm dubbed direct particle filter based distributed network localization (DiPNet). A node's location is directly estimated from the received signals, incorporating location uncertainty of neighboring nodes. The propagation effects on DiPNet become insignificant for dense networks, due to the massive-link collective physical layer processing. DiPNet achieves a near-optimal performance with low complexity, which is particularly attractive for realtime dense-network localization.
\end{abstract}

Index Terms-network localization, direct position estimation (DPE), Fisher information (FI), distributed particle filter

\section{INTRODUCTION}

Ubiquitous realtime location information in wireless networks is essential for a wide range of applications, from mass-market location-aware services like autonomous driving [1], crowd sensing [2], communication enhancement [3] and internet of things (IoT) [4], [5], to professional multi-agent collaborations such as disaster management [6], environmental sensing [7] and extraterrestrial robotic swarm exploration [8], [9]. Many of these applications are global navigation satellite system (GNSS)-impaired, which leads to challenges in localization. In urban areas, cellular networks (e.g. $3^{\text {rd }}$ Generation Partnership Project (3GPP)-long-term evolution (LTE) and the upcoming $5^{\text {th }}$ generation mobile networks (5G)) can be exploited for terrestrial localization [10]. In traditional terrestrial localization, multiple spatially separated base stations (BSs) in line-of-sight (LOS) condition to mobile terminals (MTs) are required as anchors. The multi-anchor requirements can be relaxed by jointly observing angular and distance information from large antenna arrays [11]-[13]. However, large phased arrays are not expected to be ubiquitously available in the near future, due to the space and computational limitation of the devices. For the aforementioned applications, mesh networks with high MT density are expected, where an MT directly communicates to its neighboring MTs via radio links, for example in the intelligent transport systems

S. Zhang, E. Staudinger, T. Jost, W. Wang, G. Gentner and A. Dammann are with the Institute of Communications and Navigation, German Aerospace Center (DLR), Germany.

H. Wymeersch is with the Department of Electrical Engineering, Chalmers University of Technology, Sweden.

P. A. Hoeher is with the Chair of Information and Coding Theory, University of Kiel, Germany.

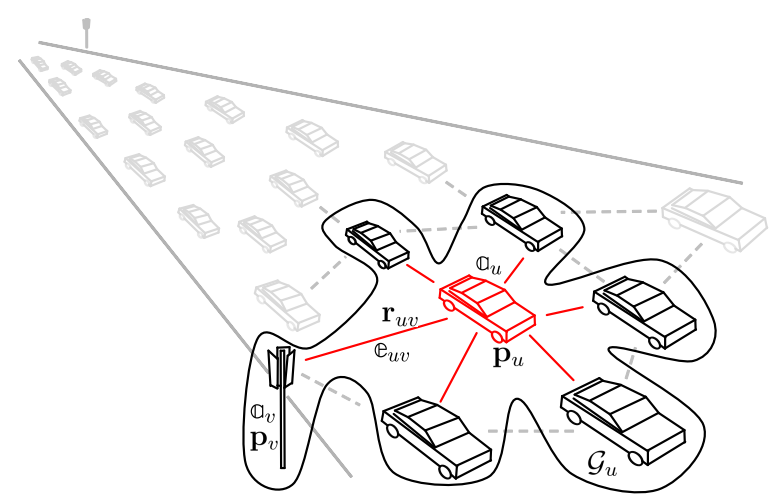

Figure 1: Network localization for cooperative vehicles.

(ITS)-G5. These massive links, often referred to as deviceto-device (D2D) links, can provide distance information between MTs and offer opportunities for network localization [4], [7], [14]-[16]. Through cooperation, an MT estimates its own location relative to the local network. The relative location information can be either fed directly to an application, e.g. autonomous vehicular platooning illustrated in Fig. 1, or fused with other sensors to obtain more precise absolute location information. Network localization possesses two unique characteristics in comparison with the traditional anchor-based localization. Firstly, a majority of the massive D2D links are in LOS condition due to short ranges. These links provide a potential of localization with high redundancy. Secondly, neighbor's location uncertainty has to be considered in addition. Intensive research has been conducted to network localization. A comprehensive overview of network localization algorithms is provided in [14], and further completed by [4]. We briefly review the algorithm classifications according to different perspectives.

\section{1) Place of Position Estimation}

An algorithm is considered as centralized if the position of agent is calculated at a fusion center [17]. Whereas if every agent calculates its own position based on local observations, the algorithm is referred to as a decentralized algorithm [14], [18].

\section{2) Model of Measurements}

Algorithms can be classified by the extractable positionrelated information, for example, signal power, carrier phase and symbol delay [19], [20]. They can also be classified according to the measurement abstraction level, whether to utilize the received waveform directly for localization, for example in direct position estimation (DPE) [21], or an 
abstracted single value metric with an associated likelihood function. For the latter case, algorithms can be further classified by the metrics extracted from the position-related information, such as time of arrival (ToA), time difference of arrival (TDoA), angle of arrival (AoA), received signal strength (RSS) [20], [22].

\section{3) Model of Unknowns}

Non-Bayesian algorithms treat unknowns as deterministic variables. A maximum likelihood (ML) approach can be implemented by least-square Gauss-Newton algorithm [20], which may suffer from local minima. Alternatively, a convex-relaxation based approach such as semi-definite programming (SDP) [23] and alternating direction method of multipliers (ADMM) [17] can be applied to reduce the effect of local minima. Bayesian algorithms treat unknowns as random variables. The main task of the algorithm is to infer the posterior probability density function (pdf) of the random variables [24]. In general, calculating the exact posterior pdf demands high dimensional marginalization, which makes it impracticable for dense networks. Kalman filter (KF)-based approaches approximate the system with linear Gaussian models, and solve it with relatively low complexity. Message passing (MP) is a popular category of Bayesian algorithms, where agents infer their posterior pdfs by only marginalizing over the inferences of their neighbors, in a recursive fashion. MP is adaptable to different system models with moderate complexity [14], [19].

\section{4) Multi-Link Fusion}

Naive algorithms such as least square (LS) treat all links identically. Measurement quality can be quantified through some characteristics of the signal, such as signal-to-noise ratio (SNR), channel impulse response (CIR), propagation condition, etc., and used in for example weighted least square (WLS) [20]. For decentralized algorithms, neighbor's position uncertainty can be taken into account in heuristic fashion [25], or systematically by the marginalization in MP. The marginalization can be realized by expectation maximization (EM), numerical integrals such as Gaussian quadrature integrals, also known as sigma points [26], or Monte Carlo (MC) integration such as used in distributed particle filtering (DPF) [27]. In [15] the impact of neighbor's position uncertainty on agent localization is quantified by the equivalent ranging information intensity (ERII). The ERII can be exploited for example by projecting the neighbor's position uncertainty onto the distance measurement [28]. This projection is also a systematic approach, which has lower complexity compared to MP.

A more exhaustive literature survey on network localization can be found in [4], [14].

In this work, we address decentralized network localization under Bayesian framework, where position information is extracted from the symbol delays. More precisely, we focus on utilizing the received waveform directly for localization, with a low complexity systematic multi-link fusion scheme.

Most of the network localization techniques apply a twostep approach, i.e. distance estimation (ranging) and location estimation (localization). In the ranging step, distance information between transmitters and receivers are extracted from the signal propagation delay in physical layer (PHY). In the localization step, the multi-link distance estimates are fused combining certain ranging error models with neighbor's location uncertainty, typically by a non-linear location estimator, for example MP such as the sum-product algorithm over a wireless network (SPAWN) [14] or its low complexity variation [28].

Unpredictable propagation conditions are a main source of ranging error, which have been intensively investigated. In LOS conditions, multipath components (MPCs) close to the LOS path may introduce a multipath bias to the distance estimate. In obstructed LOS or non-line-of-sight (NLOS) conditions, an MPC may be mistakenly considered as the LOS path, which leads to a NLOS bias. Besides, low SNR may cause a failure in LOS path detection [29], [30]. The impacts of the propagation conditions on ranging have been theoretically addressed. The Cramér-Rao bound (CRB) provides the asymptotically achievable root mean square error (RMSE) of an optimal unbiased ranging estimator, given a ranging model [31]. The Ziv-Zakai bound (ZZB) additionally takes account of the a-priori information and the detection probability and provides a tighter bound especially for low SNR scenarios [29]. In practice, a variety of ranging algorithms have been developed. In a matched filter, the distance is estimated from the strongest correlation peak, which is vulnerable to multipath effect. A first peak detection can be applied to partially mitigate the multipath effect [32]. High complexity super-resolution algorithms, e.g. space-alternating generalized expectationmaximization (SAGE) [33] and Kalman enhanced super resolution tracking (KEST) [34], iteratively estimate all MPCs for multipath mitigation. The NLOS bias effect can be mitigated by exploiting the NLOS bias distribution [30], [35], [36] or applying identify-and-discard techniques in either signal [37] or location domain [38]. Most NLOS bias mitigation techniques require a-priori information or training data and may be subject to NLOS classification failure. Recent studies propose to exploit the geometry information contained in MPCs for localization [39]-[41], which is associated with high complexity.

In two-step approaches, the location information contained in the PHY signal is not fully exploited, since the two steps are usually optimized separately. In DPE, the location information is directly extracted from the PHY processing [42], [43]. It exploits the signal correlation function as soft hypotheses and avoids point estimations for ranging. In additive white Gaussian noise (AWGN) and noncooperative scenarios, DPE has been proven to outperform the traditional two-step approaches in the presence of low SNR, which is utilized for GNSS receiver design [21], [44]. Concerning multipath scenarios, [45], [46] attempted to extend DPE to non-parametric maximum a posteriori (MAP) location estimators taking all MPCs into consideration. However, due to a high dimensional state space, it becomes computational intractable. In [47], we intuitively discussed that DPE with a one-path channel model is particularly suitable for network localization even in the presence of 
multipath. Massive LOS links collaboratively support the correct location hypothesis and reject the wrong ones with high probability.

This work is a substantial enhancement of [47] with the following contributions:

- A DiPNet algorithm is proposed, combining DPE and SPAWN for direct PHY network localization. DiPNet incorporates neighbor's location uncertainty by a low complexity equivalent measurement likelihood (EL) scheme, which enables realtime processing.

- The impacts of propagation channel on DiPNet are analytically investigated, in the sense of asymptotic localization unbiasedness and the resistance to erroneous distance information. It is proven that DiPNet is robust against unpredictable propagation effects in dense networks, due to collective PHY processing of massive links.

- Simulations with realistic channel models and two experiments are conducted, comparing DiPNet with a variety of state-of-the-art algorithms.

The remainder of the paper is organized as follows. In Section II the system model of network localization and the SPAWN algorithm are introduced. The general concept of EL and the DiPNet algorithm are derived in Section III for an orthogonal frequency-division multiplexing (OFDM) waveform. Theoretical analyses of DiPNet in multipath environments are provided in Section IV. The proposed DiPNet is evaluated compared to state-of-the-art algorithms in Section $\mathrm{V}$ by both simulations and experiments. The conclusions are drawn in Section VI.

The following notations are used throughout the paper:

- $(\cdot)^{*},(\cdot)^{T}$ and $(\cdot)^{H}$ stand for complex conjugate, transpose and conjugate transpose.

- Column vectors, matrices and sets are denoted by bold small (a), bold capital (A) and calligraphic capital letters $(\mathcal{A})$.

- I represents the identity matrix.

- Graph's elements (vertices and edges) are denoted by blackboard bold small letters ( $₫$ and $\mathbb{e}$ ).

- $\mathcal{A} / \mathbb{a}$ denotes the sub-set of $\mathcal{A}$ excluding element(s) $\mathbb{a}$.

- $\|\cdot\|$ represents the Frobenius norm of real/complexvalued scalars, vectors or matrices.

- $|\mathcal{A}|$ represents the cardinality of set $\mathcal{A}$.

- $\Re\{\cdot\}$ and $\Im\{\cdot\}$ denote the real and imaginary part(s).

- $x \sim \mathcal{N}(\boldsymbol{\mu}, \mathbf{C}), \mathcal{C N}\left(0, \sigma^{2}\right), U(a, b]$ and $\operatorname{Exp}(\lambda)$ denote random variable(s) following normal, circularlysymmetric complex normal, uniform and exponential distributions with the corresponding parameters.

- $\operatorname{Tr}[\cdot]$ denotes the trace of a matrix.

- $\mathbf{E}_{x}[\cdot]$ denotes expectation over random variable(s) $x$.

- $\nabla_{x} f, f_{x}$ and $\dot{f}$ denote the first (partial) derivative(s) of $f$.

- $\triangle_{x}^{y} f \triangleq \nabla_{y} \nabla_{x} f, f_{x y}$ and $\ddot{f}$ denote the second (partial) derivative(s) of $f$.

- $\sum_{n, m=a}^{b} \triangleq \sum_{n=a}^{b} \sum_{m=a}^{b}$ denotes double summation.

\section{Network Localization with D2D Links}

\section{A. Problem Formulation}

We consider a network, for example as illustrated in Fig. 1, composed of $|\mathcal{V}|$ nodes in two-dimensional (2D) space with node set $\mathcal{V}=\left\{\mathbb{a}_{1}, \cdots, \mathbb{a}_{\mid \mathcal{V}}\right\}$. The position vector of all nodes is defined as $\mathbf{p}=\left[\mathbf{p}_{1}^{T}, \cdots, \mathbf{p}_{|\mathcal{V}|}^{T}\right]^{T} \in \mathbb{R}^{2|\mathcal{V}|}$, where $\mathbf{p}_{u}=\left[x_{u}, y_{u}\right]^{T}$ are the 2D Cartesian coordinates of node $\mathbb{a}_{u}$. Out of the $|\mathcal{V}|$ nodes, $|\mathcal{B}|$ are anchors with known positions $\mathbf{p}_{\mathcal{B}} \in \mathbb{R}^{2|\mathcal{B}|}$, and belong to the anchor set $\mathcal{B}$. The remaining $|\mathcal{M}|$ nodes are agents, which form the agent set $\mathcal{M}$. Agents estimate their position $\mathbf{p}_{\mathcal{M}} \in \mathbb{R}^{2|\mathcal{M}|}$ w.r.t. the anchors in the anchor-based case and w.r.t. the other agents in the anchor-free case. Nodes which can communicate and conduct measurements with $\mathbb{a}_{u}$ via radio signals are considered as neighbors of $\mathbb{a}_{u}$ and included in its neighbor set $\mathcal{V}_{u}$. If the neighbor is an agent, it is also included in the neighboring agent set $\mathcal{M}_{u}$ of $\mathbb{a}_{u}$. We assume a symmetric neighborhood relationship, i.e. $\mathbb{a}_{v}$ is a neighbor of $\mathbb{a}_{u}$, if and only if $\mathbb{a}_{u}$ is also a neighbor of $\mathbb{a}_{v}$. The link between these two nodes is denoted as $\mathbb{e}_{u v}$. Radio signals transmitted from $\mathbb{a}_{v}$ and received by $\mathbb{a}_{u}$ are denoted as $\mathbf{r}_{u v}$, containing relative position information between the two nodes, for example the inter-node distance $d_{u v, 0}$. Agent $\mathbb{a}_{u}$ extracts generic position-related measurements $\mathbf{z}_{u v}$ from $\mathbf{r}_{u v}$. The total link set is defined as $\mathcal{E}_{0}=\left\{\cdots, \mathbb{e}_{u v}, \cdots\right\}$, with all measurements $\mathbf{z}=\left[\cdots, \mathbf{z}_{u v}^{T}, \cdots\right]^{T}$, for all neighboring pairs $\left(\mathbb{a}_{u}, \mathbb{a}_{v}\right)$. We utilize graph theory to generally formulate the network localization problem, including both anchorbased and anchor-free cases. The network can be interpreted as a framework $\mathcal{F}_{0}=\left(\mathcal{G}_{0}, \mathbf{p}\right)$ with an underlying undirected graph $\mathcal{G}_{0}=\left(\mathcal{V}, \mathcal{E}_{0}\right)$, where nodes are interpreted as the vertices and measurement links as edges. In order to incorporate the anchors, we extend $\mathcal{E}_{0}$ with virtual links without measurement to completely connect all the anchors, i.e. the new edge set is $\mathcal{E}=\mathcal{E}_{0} \bigcup\left\{\cdots, \mathbb{e}_{u v}, \cdots\right\}$, for all anchor pairs $\left(\mathbb{a}_{u}, \mathbb{a}_{v}\right)$. The extended graph and framework become $\mathcal{G}=(\mathcal{V}, \mathcal{E})$ and $\mathcal{F}=(\mathcal{G}, \mathbf{p})$, respectively. In the case of insufficient number of anchors, the position of an agent is not observable. Therefore, only the 'shape' of the network can be estimated. The framework can be estimated up to rigid affine transformation $\mathcal{T}(\hat{\mathbf{p}})$ including translation, rotation and flipping, where $\hat{\mathbf{p}}$ is the estimated position vector of nodes. The objective of network localization is to find a framework $\hat{\mathcal{F}}=(\mathcal{G}, \mathbf{q})$, with nodes' coordinates $\mathbf{q}$, whose 'shape' is as 'similar' as possible to the original one $\mathcal{F}$, given all the observations $\mathbf{z}$. We define the average shape difference $\varepsilon_{\mathbf{q}, \mathbf{p}}$

$$
\varepsilon_{\mathbf{q}, \mathbf{p}} \triangleq \sqrt{\frac{1}{|\mathcal{M}|}\left\|\mathcal{T}_{\text {opt }}(\mathbf{q})-\mathbf{p}\right\|^{2}},
$$

as the metric to assess the 'similarity' of these two frameworks, where $\mathcal{T}_{\text {opt }}$ is the optimal affine transformation [48], fixing the anchor's transformed estimates to their true posi- 
tion. The network localization problem can be stated as

$$
\begin{array}{ll} 
& \hat{\mathbf{p}}=\underset{\mathbf{q}}{\arg \min } \varepsilon_{\mathbf{q}, \mathbf{p}} \\
\text { s.t. } & \mathcal{T}_{\text {opt }}\left(\hat{\mathbf{p}}_{\mathcal{B}}\right)=\mathbf{p}_{\mathcal{B}} .
\end{array}
$$

The network localizability is described in the rigidity theory [49], which is beyond the scope of this paper. In this paper, we assume $\mathcal{F}$ is always globally rigid. Another indirect metric, which is used in Section $\mathrm{V}$ to evaluate the performance of network localization, is the framework distance RMSE

$$
\varepsilon_{\mathbf{d}}=\sqrt{\frac{\sum_{\forall \Phi_{u} \in \mathcal{M}, \Phi_{v} \in \mathcal{V} / \Phi_{u}} \varepsilon_{u v}^{2}}{|\mathcal{M}|(|\mathcal{V}|-1)}}
$$

where $\varepsilon_{u v}=\left\|\hat{d}_{u v}-d_{u v, 0}\right\|$ is the absolute error of framework distance estimate $\hat{d}_{u v}=\left\|\hat{\mathbf{p}}_{u}-\hat{\mathbf{q}}_{v}\right\|$.

\section{B. OFDM Waveform and Multipath Propagation}

We assume the signal $\mathbf{r}_{u v}$ is modulated with the OFDM scheme, which is widely employed in communications, e.g. in wireless local area network (WLAN), LTE and ITSG5, as well as foreseen in 5G. An OFDM signal $s_{u v}(t)$ is transmitted from $\mathbb{a}_{v}$ and received by $\mathbb{a}_{u}$ through link $\mathbb{e}_{u v}$. We assume an odd number $N$ of subcarriers, without loss of generality. The transmitted OFDM symbol is expressed as

$$
s_{u v}(t)=\frac{1}{\sqrt{N}} \sum_{n=-\frac{N-1}{2}}^{\frac{N-1}{2}} S_{n} e^{j \omega n t},
$$

where $\omega=2 \pi f_{s c}, f_{\mathrm{sc}}$ is the subcarrier spacing, $n$ is the subcarrier index, and $S_{n}$ is the information symbol carried by the $n^{\text {th }}$ subcarrier. In a realistic scenario, the signal is not only distorted by sensor noise, but also affected by the propagation channel. For LOS scenarios, the signal propagates along the LOS path and some additional paths, referred to as MPCs. Whereas for NLOS scenarios, the signal is solely received via the MPCs. A generic path component $l$

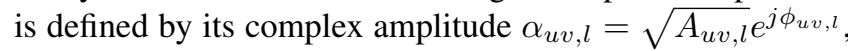
with a power $A_{u v, l}$ and a phase $\phi_{u v, l}$, and the total propagation delay $\tau_{u v, l}=\tau_{u v, 0}+\delta_{u v, l}+b_{u v}$, which includes the LOS delay $\tau_{u v, 0}=d_{u v, 0} / c_{0}$ with the propagation speed $c_{0}$, the NLOS delay $b_{u v}$ and the path's delay additional to the potential LOS path $\delta_{l}$. The NLOS delay $b_{u v}$ is positive for NLOS scenarios and zero for LOS scenarios. The LOS path is denoted with index 0 , i.e. $\delta_{u v, 0}=0$. NLOS scenarios are included by setting $\alpha_{u v, 0}=0$. The received sampled signal can be generally written as the superposition of the potential LOS path and $L$ MPCs distorted with the additive Gaussian noise $\epsilon_{u v}(i T) \sim \mathcal{C N}\left(0, \sigma_{u}^{2}\right)$ as

$$
r_{u v}(i T)=\sum_{l=0}^{L} \alpha_{u v, l} s_{u v, l}\left(i T-\tau_{u v, l}\right)+\epsilon_{u v}(i T),
$$

with a sampling period $T$ and $\forall i=1, \cdots, N$. The (delayed) signal samples are represented in vector forms, for example, $\mathbf{r} \triangleq[r(T), \cdots, r(N T)]^{T}$ and $\mathbf{s}(\tau) \triangleq[s(T-\tau), \cdots, s(N T-$ $\tau)]^{T}$. The clock offsets between nodes may bias the delaybased distance information. However, it can be eliminated with multi-way ranging. For the AWGN case, the two-way ranging with an amplify-and-forward scheme [50] is equivalent to the synchronized one-way ranging [51]. For the multipath scenario, it is analogous to the synchronized oneway ranging, with a channel equivalent to the convolution of the forward and backward channels.

\section{Distributed Bayesian Network Localization}

A Bayesian estimator treats positions $\mathbf{p}$ as random variables and estimates them from the a posteriori $\operatorname{pdf} p(\mathbf{p} \mid \mathbf{z})$. The $a$ posteriori pdf incorporates the $a$ priori $\operatorname{pdf} p(\mathbf{p})$ and the observation likelihood function $p(\mathbf{z} \mid \mathbf{p})$ by Bayes' rule according to

$$
p(\mathbf{p} \mid \mathbf{z}) \propto p(\mathbf{p}) p(\mathbf{z} \mid \mathbf{p}) .
$$

We utilize the Bayesian estimation framework for description simplicity. With a state transition model and the firstorder Markov model assumption, the framework can be extended to recursive Bayesian tracking with sequential measurements. Particularly for dense networks, a distributed localization algorithm is often advantageous, where an agent $\mathbb{a}_{u}$ estimates its own position using the marginalized $a$ posteriori $\operatorname{pdf} p\left(\mathbf{p}_{u} \mid \mathbf{z}\right)$

$$
\begin{aligned}
p\left(\mathbf{p}_{u} \mid \mathbf{z}\right) & \propto p\left(\mathbf{p}_{u}\right) \int p\left(\mathbf{p}_{\mathcal{V} / \Phi_{u}} \mid \mathbf{p}_{u}\right) \\
& \times \prod_{\forall \in_{v w} \in \mathcal{E}_{0}} p\left(\mathbf{z}_{v w} \mid \mathbf{p}_{v}, \mathbf{p}_{w}\right) \mathrm{d} \mathbf{p}_{\mathcal{V} / \Phi_{u}} .
\end{aligned}
$$

Hereafter, the true position of an agent $\mathbb{a}_{u}$ is denoted as $\mathbf{p}_{u, 0}$, in order to differentiate from the unknown random variable $\mathbf{p}_{u}$. We consider anchors as special agents with Dirac $a$ priori pdfs at their true positions. Due to cooperation among agents, a $2(|\mathcal{M}|-1)$ dimensional integral is needed for an exact distributed Bayesian estimator of $\mathbf{p}_{u}$, which makes it impracticable. belief propagation (BP) is a popular approach to reduce the complexity of marginalization, employed for example in the SPAWN algorithm [14]. In SPAWN, an agent $\mathbb{a}_{u}$ only considers a local sub-framework $\mathcal{F}_{u}=\left(\mathcal{G}_{u}, \mathbf{p}_{\mathcal{N}_{u}}\right)$ with a extended node set $\mathcal{N}_{u}=\mathcal{V}_{u} \cup\left\{\mathbb{a}_{u}\right\}$ and the underlying star subgraph $\mathcal{G}_{u}=\left(\mathcal{N}_{u}, \mathcal{E}_{u}\right)$, for example illustrated in Fig. 1. The edge set $\mathcal{E}_{u}$ contains all the links between $\mathbb{a}_{u}$ and its neighbors. The marginalized a posteriori $\operatorname{pdf} p\left(\mathbf{p}_{u} \mid \mathbf{z}\right)$ is approximated by the belief $b_{u}^{(K)}$ of agent $\mathbb{a}_{u}$, which is updated by exchanging belief information with neighbors for $K$ algorithmic iterations

$$
b_{u}^{(k)}=b_{u}^{(0)} \prod_{\forall a_{v} \in \mathcal{V}_{u}} \int b_{v}^{(k-1)} p\left(\mathbf{z}_{u v} \mid \mathbf{p}_{u}, \mathbf{p}_{v}\right) \mathrm{d} \mathbf{p}_{v},
$$

where $k=1, \cdots, K$. The SPAWN reduces the complexity to $\left|\mathcal{M}_{u}\right|$ integrals with four dimensions for each iteration. The marginalization in (9) can be realized by Monte Carlo integration as in non-parametric belief propagation (NBP) [18], by numerical integration for example in cubature belief propagation (CBP) [26], [52], or analytically with parametric belief propagation (PBP) for special distributions [53]. Neither CBP nor PBP is suitable for direct network localization, since the process of extracting location in- 
formation from PHY signal is highly non-linear and nonGaussian. In this case NBP is favorable. However, propagating non-parametric beliefs to neighbors is considered highly inefficient due to high communication throughput demands. Besides, the Monte Carlo integration requires a high computational power [53]. In our proposed approach, non-parametric belief is updated locally at agents. Only the first two moments of the belief are broadcast to the neighbors, in order to reduce the communication overhead.

\section{LOW-COMPLEXITY NETWORK LOCALIZATION}

\section{A. Equivalent Measurement Likelihood (EL)}

To further reduce the complexity, we define an EL based on Fisher information (FI) theory, which will be used later for the proposed DiPNet algorithm. The joint pdf $q\left(\mathbf{p}_{\mathcal{N}_{u}}, \mathbf{z}_{\mathcal{E}_{u}}\right)$ of the simplified graph $\mathcal{G}_{u}$ can be written as

$$
q\left(\mathbf{p}_{\mathcal{N}_{u}}, \mathbf{z}_{\mathcal{E}_{u}}\right)=b_{u}^{(0)} \prod_{\mathbb{a}_{v} \in \mathcal{V}_{u}} b_{v}^{(k-1)} p\left(\mathbf{z}_{u v} \mid \mathbf{p}_{u}, \mathbf{p}_{v}\right) .
$$

The Bayesian information matrix (BIM) $\mathbf{J}_{\mathcal{N}_{u}}$ of $\mathbf{p}_{\mathcal{N}_{u}}$ is expressed as

$$
\mathbf{J}_{\mathcal{N}_{u}}=\mathbf{I}_{\mathcal{N}_{u}}+\mathbf{E}_{\mathbf{p}_{\mathcal{N}_{u}}, \mathbf{z}_{\mathcal{E}_{u}}}\left[\triangle_{\mathbf{p}_{\mathcal{N}_{u} u}}^{\mathbf{p}_{\mathcal{N}_{u}}} \ln p\left(\mathbf{z}_{\mathcal{E}_{u}} \mid \mathbf{p}_{\mathcal{N}_{u}}\right)\right],
$$

where $\mathbf{I}_{\mathcal{N}_{u}}=\operatorname{diag}\left[\mathbf{I}_{u}^{(0)}, \cdots, \mathbf{I}_{v}^{(k-1)}, \cdots\right], \forall \mathbb{a}_{v} \in \mathcal{V}_{u}$ is the $a$ priori location information of $\mathbf{p}_{\mathcal{N}_{u}}$, with individual a priori information $\mathbf{I}_{w}^{(k)}$ defined as

$$
\mathbf{I}_{w}^{(k)} \triangleq-\mathbf{E}_{\mathbf{p}_{w}}\left[\triangle_{\mathbf{p}_{w}}^{\mathbf{p}_{w}} \ln b_{w}^{(k)}\right] .
$$

The equivalent Bayesian information matrix (EBIM) $\mathbf{J}_{u}$ of $\mathbf{p}_{u}$, derived from the theory of Schur's complement [54], determines the best achievable variance for the a posteriori estimate $\hat{\mathbf{p}}_{u}$. Assuming the beliefs $b_{w}^{(k)}$ are concentrated at their a priori means $\overline{\mathbf{p}}_{w}=\mathbf{E}_{\mathbf{p}_{w}}\left[\mathbf{p}_{w}\right]$, the EBIM is approximated as

$$
\mathbf{J}_{u}=\mathbf{I}_{u}^{(0)}+\sum_{v \in \mathcal{V}_{u}} \tilde{\nu}_{u v} \overline{\mathbf{e}}_{u v} \overline{\mathbf{e}}_{u v}^{T},
$$

where $\tilde{\nu}_{u v}$ is the ERII defined as

$$
\tilde{\nu}_{u v}=\frac{\bar{\nu}_{u v}}{1+\bar{\nu}_{u v} \sigma_{v \mapsto u v}^{2}} .
$$

The projection vector $\overline{\mathbf{e}}_{u v}$ and the projected variance $\sigma_{v \mapsto u v}^{2}$ are defined as

$$
\overline{\mathbf{e}}_{u v} \triangleq \frac{\left(\overline{\mathbf{p}}_{u}-\overline{\mathbf{p}}_{v}\right)}{\left\|\overline{\mathbf{p}}_{u}-\overline{\mathbf{p}}_{v}\right\|}, \quad \sigma_{v \mapsto u v}^{2} \triangleq \overline{\mathbf{e}}_{u v}^{T}\left(\mathbf{I}_{v}^{(k-1)}\right)^{-1} \overline{\mathbf{e}}_{u v}
$$

which project the location uncertainty of $a_{v}$ onto the measurement link $e_{u v}$. The ranging information intensity (RII) $\bar{\nu}_{u v}$ is defined as

$$
\bar{\nu}_{u v} \triangleq-\mathbf{E}_{\mathbf{p}_{u}, \mathbf{p}_{v}}[\underbrace{\mathbf{E}_{\mathbf{z}_{u v} \mid \mathbf{p}_{u}, \mathbf{p}_{v}}\left[\frac{\mathrm{d}^{2} \ln p\left(\mathbf{z}_{u v} \mid \mathbf{p}_{u}-\mathbf{p}_{v}\right)}{\mathrm{d} d_{u v}^{2}}\right]}_{\triangleq \nu_{u v}}],
$$

where $d_{u v}=\left\|\mathbf{p}_{u}-\mathbf{p}_{v}\right\|$. The proof of (13) is detailed in Appendix I. A similar result has been reported in [15]. The EBIM in (13) has a similar expression as the location infor- mation $\mathbf{J}_{(u, u)}$ assuming the neighbor's position is perfectly known

$$
\mathbf{J}_{(u, u)}=\mathbf{I}_{u}^{(0)}+\mathbf{E}_{\mathbf{p}_{\mathcal{N}_{u}}}\left[\sum_{v \in \mathcal{V}_{u}} \nu_{u v} \mathbf{e}_{u v} \mathbf{e}_{u v}^{T}\right]
$$

where $\mathbf{e}_{u v} \triangleq \nabla_{\mathbf{p}_{u}} d_{u v}$ is the direction vector. Alternatively, equation (14) can be expressed by the equivalent ranging uncertainty

$$
\tilde{\nu}_{u v}^{-1}=\bar{\nu}_{u v}^{-1}+\sigma_{v \mapsto u v}^{2} .
$$

Hence the neighbor's position uncertainty can be additively aggregated to the ranging uncertainty. We utilize these observations to define an EL, which can be applied to further reduce the complexity of network localization.

Definition 1. An equivalent measurement likelihood (EL) $\tilde{p}\left(\mathbf{z}_{u v} \mid \mathbf{p}_{u}, \mathbf{p}_{v}\right)$ is a pdf of $\mathbf{z}_{u v}$ conditioning on $\mathbf{p}_{u}$ and $\mathbf{p}_{v}$, such that

$$
-\mathbf{E}_{\mathbf{z}_{u v} \mid \mathbf{p}_{u}, \mathbf{p}_{v}}\left[\frac{d^{2} \ln \tilde{p}\left(\mathbf{z}_{u v} \mid \mathbf{p}_{u}, \mathbf{p}_{v}\right)}{d d_{u v}^{2}}\right]=\tilde{\nu}_{u v} .
$$

The system can be further simplified to non-cooperative localization, with a virtual likelihood function modeled with the EL and the neighbor's virtual a priori pdf modeled as a Dirac function at point $\hat{\mathbf{p}}_{v}^{(k-1)}$. The EBIM of the simplified system $\tilde{\mathbf{J}}_{u}$ equals to the original $\mathbf{J}_{u}$. Low-complexity distributed network localization algorithms can be designed as follows. Agent $a_{u}$ receives the first two moments of its neighbor's non-parametric belief, namely the position estimate $\hat{\mathbf{p}}_{v}^{(k-1)}$ and the covariance estimate $\operatorname{cov}\left[\hat{\mathbf{p}}_{v}^{(k-1)}\right]$, to approximate ERII in (14). Instead of the sum-product algorithm in (9), the belief can be updated by the simplified model using the EL

$$
b_{u}^{(k)} \approx b_{u}^{(0)} \prod_{\forall \mathbb{a}_{v} \in \mathcal{V}_{u}} \tilde{p}\left(\mathbf{z}_{u v} \mid \mathbf{p}_{u}, \hat{\mathbf{p}}_{v}^{(k-1)}\right) .
$$

The EL-based algorithm further reduces the complexity to a single 2-dimensional integral per algorithmic iteration, which enables distributed Bayesian network localization in realtime. The concept of EL can be generally applied to any distance-based measurement models. For example in [28], it is used in two-step network localization with Gaussian ranging models by exploiting the equivalent ranging variances (ERVs). In Section III-B, we introduce the DiPNet algorithm, where an EL is adapted for direct localization from the OFDM waveform.

\section{B. DiPNet with OFDM Waveform}

The DiPNet algorithm is derived based on a one-path received signal assumption in LOS condition, i.e. $\left\|\alpha_{u v, 0}\right\| \neq 0$ and $L=0$. The assumed received signal is denoted as $\mathbf{r}_{u v, 0}$, in order to be distinguished from the true received signal $\mathbf{r}_{u v}$. The one-path signal model enables low complexity location estimation, at the cost of sub-optimality due to model mismatch. In two step approaches, this mismatch may lead to erroneous location estimates. In Sections IV and V it is discussed that the impacts of the model mismatch on the proposed DiPNet algorithm become insignificant in dense 
networks, as a result of collective PHY processing. The one-path model can be described by the likelihood function $p\left(\mathbf{r}_{u v, 0} \mid \mathbf{p}_{u}, \mathbf{p}_{v}, \alpha_{u v, 0}\right)$. The amplitude $\alpha_{u v, 0}$ is irrelevant to position estimate and estimated separately as

$$
\hat{\alpha}_{u v, 0}=\frac{\mathbf{s}_{u v}\left(\left\|\mathbf{p}_{u}-\mathbf{p}_{v}\right\| / c_{0}\right)^{H} \mathbf{r}_{u v, 0}}{\left\|\mathbf{s}_{u v}\left(\left\|\mathbf{p}_{u}-\mathbf{p}_{v}\right\| / c_{0}\right)\right\|^{2}},
$$

with a constant denominator expressed as $\left\|\mathbf{s}_{u v}\right\|^{2}$. According to the theory of separable variables [55], the ranging likelihood function can be expressed by inserting the phase estimate into the original likelihood function as

$$
p\left(\mathbf{r}_{u v, 0} \mid \mathbf{p}_{u},-\mathbf{p}_{v}\right) \propto \exp \left(\frac{\left\|f\left(\left\|\mathbf{p}_{u}-\mathbf{p}_{v}\right\|\right)\right\|^{2}}{\sigma_{u}^{2}\left\|\mathbf{s}_{u v}\right\|^{2}}\right) .
$$

The cross-correlation function $f\left(\left\|\mathbf{p}_{u}-\mathbf{p}_{v}\right\|\right)$ is written as

$$
f\left(\left\|\mathbf{p}_{u}-\mathbf{p}_{v}\right\|\right)=\mathbf{r}_{u v, 0}^{H} \mathbf{s}_{u v}\left(\left\|\mathbf{p}_{u}-\mathbf{p}_{v}\right\| / c_{0}\right) .
$$

The logarithmic likelihood function is proportional to the squared cross-correlation function (SCF) $\left\|f\left(\left\|\mathbf{p}_{u}-\mathbf{p}_{v}\right\|\right)\right\|^{2}$, which is asymptotically maximized at $\left\|\mathbf{p}_{u}-\mathbf{p}_{v}\right\|=d_{u v, 0}$. We will use these properties in Section IV to evaluate the performance of DiPNet in multipath propagation conditions. The ranging uncertainty $\bar{\nu}_{u v}^{-1}$ in (18), i.e. the ranging CRB denoted by $\mathrm{CRB}_{u v}$, is derived as in [28] with the one-path OFDM signal model as

$$
\bar{\nu}_{u v}^{-1}=\mathrm{CRB}_{u v}=\frac{c_{0}^{2} \sigma_{u}^{2}}{2\left\|\hat{\alpha}_{u v, 0}\right\|^{2} \omega^{2} \sum_{n=-\frac{N-1}{2}}^{\frac{N-1}{2}}\left\|S_{n}\right\|^{2} n^{2}} .
$$

A choice of EL for DiPNet is to aggregate the neighbor's position uncertainty as noise, i.e.

$$
\tilde{p}\left(\mathbf{r}_{u v, 0} \mid \mathbf{p}_{u}, \hat{\mathbf{p}}_{v}^{(k-1)}\right) \propto \exp \left(\frac{\left\|f\left(\left\|\mathbf{p}_{u}-\hat{\mathbf{p}}_{v}^{(k-1)}\right\|\right)\right\|^{2}}{\tilde{\sigma}_{u v}^{2}\left\|\mathbf{s}_{u v}\right\|^{2}}\right),
$$

where $\tilde{\sigma}_{u v}^{2}$ is the equivalent noise variance (ENV). The corresponding equivalent ranging uncertainty $\tilde{\nu}_{u v}^{-1}$ has an expression similar to $\bar{\nu}_{u v}^{-1}$ in (24), by replacing $\sigma_{u}^{2}$ with $\tilde{\sigma}_{u v}^{2}$. The ENV is derived by inserting $\bar{\nu}_{u v}^{-1}$ and $\tilde{\nu}_{u v}^{-1}$ into (18) as

$$
\tilde{\sigma}_{u v}^{2}=\sigma_{u}^{2}+\frac{2 \omega^{2} \sigma_{v \mapsto u v}^{2}}{c_{0}^{2}}\left\|\hat{\alpha}_{u v, 0}\right\|^{2} \sum_{n=-\frac{N-1}{2}}^{\frac{N-1}{2}}\left\|S_{n}\right\|^{2} n^{2} .
$$

In the proposed DiPNet, the location belief is updated according to (20) and (25), by replacing the algorithmic signal model $\tilde{\mathbf{r}}_{u v}$ with the real received signal $\mathbf{r}_{u v}$. A DPF is implemented at each agent for non-parametric belief calculation [27], [28]. $Q$ particles $\mathcal{P}_{u}^{(0)}=\left\{P_{u}^{(1,0)}, \cdots, P_{u}^{(Q, 0)}\right\}$ are drawn at $\mathbb{a}_{u}$ according to its a priori pdf. Each particle $P_{u}^{(p, 0)}=\left(\mathbf{p}_{u}^{(p, 0)}, w_{u}^{(p, 0)}\right)$ is defined with its position $\mathbf{p}_{u}^{(p, 0)}$ and a normalized weight $w_{u}^{(p, 0)}$. The non-parametric belief at $k^{\text {th }}$ iteration can be represented as

$$
b_{u}^{(k)} \approx \sum_{p=1}^{Q} w_{u}^{(p, k)} \delta\left(\mathbf{p}_{u}-\mathbf{p}_{u}^{(p, 0)}\right)
$$

The weight is updated by (20) as

$$
w_{u}^{(p, k)}=\frac{w_{u}^{(p, 0)}}{C_{u}} \prod_{v \in \mathcal{V}_{u}} \tilde{p}\left(\mathbf{r}_{u v} \mid \mathbf{p}_{u}^{(p, 0)}, \hat{\mathbf{p}}_{v}^{(k-1)}\right),
$$

where $C_{u}$ is the normalization factor. With all building blocks been introduced, we can finally describe the overall DiPNet algorithm for an agent $a_{u}$ in Algorithm 1. For numerical stability, DiPNet is operated in logarithm domain with Jacobian algorithm as described in [56].

DiPNet only requests evaluating cross-correlation function $f\left(\left\|\mathbf{p}_{u}^{(p, 0)}-\hat{\mathbf{p}}_{v}^{(k-1)}\right\|\right)$ at $Q$ discrete points. An efficient interpolation technique, for example the inverse fast Fourier transform (IFFT), can be applied to calculate these values. An advanced method can be utilized to further reduce the computational complexity of interpolation [57]. DiPNet has a complexity comparable to the ranging step in twostep approaches, where the evaluation of cross-correlation function $f\left(d_{u v}\right)$ is also required.

\section{DiPNet vs. Traditional Network Localization Algorithms}

A complexity and communication overhead comparison of different algorithms is summarized in Table I, where $K_{\tau}$ and $K_{s}$ are the number of iterations for delay estimation and SAGE, respectively, and $(L+1)$ is the model order in SAGE. The DiPNet composed of EL and $\beta$-interpolated IFFT requires only few messages in the order of $\left|\mathcal{M}_{u}\right| K$ to transmit and computational complexity nearly linear to the number of particles $Q$ and the number of samples $N$.

In comparison to the state-of-the-art network localization algorithms mentioned in the introduction, the DiPNet utilizes a low measurement abstraction level as in DPE. It adapts the Bayesian framework of MP to enable decentralized calculation. Unlike traditional MP, neighbor's belief is exploited to calculate the EL instead of marginalization, to achieve a flexible belief inference while retaining low complexity. The concept of DiPNet even shares some commonality to the vector tracking algorithm of GNSS receivers, where the positioning solution feeds back into the tracking of signal to reject outliers [58].

Compared with the traditional two-step approach, the direct localization approach applied in DiPNet has also a few unfavorable properties. Firstly, the cross correlation function of each link has to be stored in a lookup table, which requires more memory, or communication overhead for a centralized variant, and has limited resolution. Secondly, in order to apply low complexity MP algorithms, for example by numerical integral [26], the measurement message has to possess certain properties, which is not fulfilled by the direct localization approach. Hence, it is not straightforward to extend direct localization to low complexity MP.

We first prove the DiPNet is more robust in the considered multipath environments, in comparison with the traditional algorithms in the next Section. Then in Section $\mathrm{V}$ we verify with simulation and experimental results, that for the considered applications, the drawbacks of DiPNet are insignificant compared to its advantages over the traditional algorithms. 


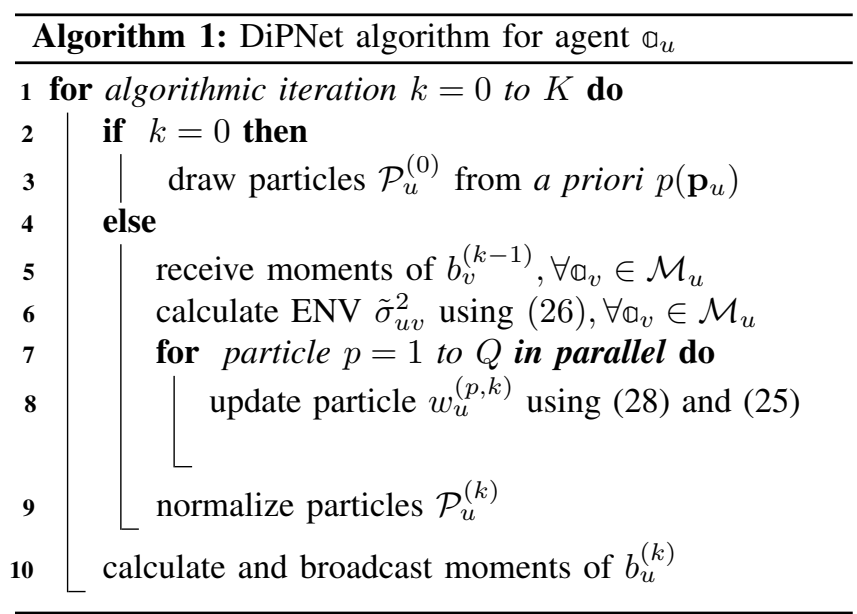

Table I: Comparison of algorithms in the sense of complexity and transmitted messages

\begin{tabular}{|c|c|c|c|c|}
\hline \multicolumn{3}{|c|}{ Algorithm } & Complexity & Messages \\
\hline $\begin{array}{l}\text { Position } \\
\text { (per agent) }\end{array}$ & \multicolumn{2}{|c|}{$\begin{array}{c}\text { Exact } \\
\text { Sampled SPAWN } \\
\text { EL }\end{array}$} & $\begin{array}{l}Q^{|\mathcal{M}|} \\
Q^{2}\left|\mathcal{M}_{u}\right| K \\
Q\left|\mathcal{V}_{u}\right| K\end{array}$ & $\begin{array}{l}Q|\mathcal{M}| \\
Q\left|\mathcal{M}_{u}\right| K \\
\left|\mathcal{M}_{u}\right| K\end{array}$ \\
\hline \multirow{2}{*}{$\begin{array}{c}\text { Signal } \\
\text { (per link) }\end{array}$} & Ranging & $\begin{array}{c}\text { Correlation } \\
\text { SAGE }\end{array}$ & \multirow{2}{*}{$\begin{array}{l}K_{\tau} N \\
K_{s}(L+1) K_{\tau} N \\
\beta N \log \beta N\end{array}$} & \\
\hline & $\beta$-inter & olated IFFT & & \\
\hline \multicolumn{3}{|c|}{ DiPNet (per agent) } & $\begin{array}{l}Q\left|\mathcal{V}_{u}\right| K+ \\
\left|\mathcal{V}_{u}\right| \beta N \log \beta N\end{array}$ & $\left|\mathcal{M}_{u}\right| K$ \\
\hline
\end{tabular}

\section{DiPNet in Multipath Environments}

The multipath propagation condition violates the onepath signal model assumed in DiPNet, which leads to a sub-optimality for localization. Due to the stochastic realizations of network and channel, it is difficult to analyze the impacts of the model mismatch from a single link on the DiPNet performance. Instead, we investigate the collective propagation impacts from all links, utilizing the fact that a generic agent $\mathbb{a}_{u}$ in a dense network is often connected with a large number of neighbors. We consider an agent $\mathbb{a}_{u}$ surrounded by $\left|\mathcal{V}_{u}\right|$ neighboring anchors. The agent $\mathbb{a}_{u}$ has a uniformly distributed a priori belief of its location $b_{u}^{(0)}$ and, updates its belief to $b_{u}^{(1)}$ by the received signals $\mathbf{r}_{u v}, \forall \mathbb{a}_{v} \in \mathcal{V}_{u}$. As mentioned in Section III, the updated logarithmic belief can be expressed with the summation of the SCFs of all links as

$$
\log b_{u}^{(1)} \propto \sum_{\mathbb{a}_{v} \in \mathcal{V}_{u}}\left\|\mathbf{r}_{u v}^{H} \mathbf{s}_{u v}\left(\left\|\mathbf{p}_{u}-\mathbf{p}_{v}\right\| / c_{0}\right)\right\|^{2} .
$$

For discussion convenience, we define a polar coordinate system originates at the true position $\mathbf{p}_{u, 0}$ of $\mathbb{a}_{u}$, whose axes are aligned with the ones of original Cartesian coordinate system. The position of $\mathbb{a}_{v}$ is reformulated with the LOS distance $d_{u v, 0}$ and the angle $\theta_{u v}$ to $\mathbb{a}_{u}$ as

$$
\mathbf{p}_{v}=\mathbf{p}_{u, 0}+d_{u v, 0}\left[\cos \theta_{u v}, \sin \theta_{u v}\right]^{T} \text {. }
$$

The position of $a_{u}$, which is apart from the true position with a distance $\triangle d=\triangle \tau c_{0}$ and an angle $\tilde{\theta}$, is expressed as

$$
\mathbf{p}_{u}=\mathbf{p}_{u, 0}+\Delta d[\cos \tilde{\theta}, \sin \tilde{\theta}]^{T} .
$$

The distance $\left\|\mathbf{p}_{u}-\mathbf{p}_{v}\right\|$ is reformulated as

$$
\left\|\mathbf{p}_{u}-\mathbf{p}_{v}\right\|=\sqrt{d_{u v, 0}^{2}+\Delta d^{2}-2 d_{u v, 0} \Delta d \cos \left(\theta_{u v}-\tilde{\theta}\right)} .
$$

We have in addition the following statistical assumptions of the link parameters:

- The angle of the neighbor is uniformly independent and identically distributed (i.i.d.) around $\mathbf{p}_{u, 0}$, i.e. $\theta_{u v} \sim$ $U[0,2 \pi)$;

- The LOS distance $d_{u v, 0}$ between $\mathbb{a}_{u}$ and $\mathbb{a}_{v}$ is i.i.d. and independent from $\theta_{u v}$;

- The link's LOS/NLOS condition $X_{u v} \in\{$ LOS, NLOS $\}$ is i.i.d. given $d_{u v, 0}$;

- The number of MPCs $L_{u v}$ of each link is i.i.d. given $X_{u v}$;

- The amplitude of each path $\alpha_{u v, l}$ is i.i.d., with a power $A_{u v, l}$ depending on $X_{u v}$ and $d_{u v, 0}$ and a uniformly distributed phase $\phi_{u v, l} \sim U[0,2 \pi)$.

- The NLOS delay and additional path delay of MPCs, $b_{u v}$ and $\delta_{u v, l}$, are i.i.d. given $X_{u v}$.

Since the propagation parameters of all links are i.i.d., in an asymptotic case where $\left|\mathcal{V}_{u}\right| \rightarrow \infty$, we have

$$
\log b_{u}^{(1)} \rightarrow\left|\mathcal{V}_{u}\right| \mathbf{E}_{\mathbf{x}_{u v}}\left[\left\|\mathbf{r}_{u v}^{H} \mathbf{s}_{u v}\left(\left\|\mathbf{p}_{u}-\mathbf{p}_{v}\right\| / c_{0}\right)\right\|^{2}\right],
$$

where $\mathbf{x}_{u v}$ is the random variables of the link $\mathbb{e}_{u v}$, including all parameters mentioned above and the noise $\boldsymbol{\epsilon}_{u v}$. Hereafter, the subscripts $u$ and $v$ are omitted for simplicity when a single link $\mathbb{e}_{u v}$ is under investigation. The joint pdf of the link's random variables can be factorized as

$$
\begin{aligned}
p(\mathbf{x}) & =p\left(d_{0}\right) p(\theta) p(\boldsymbol{\epsilon}) \operatorname{Pr}\left(X=\chi \mid d_{0}\right) p(b ; \chi) p\left(A_{0} \mid d_{0} ; \chi\right) \\
& \times p\left(\phi_{0}\right) \operatorname{Pr}(L=\tilde{L} ; \chi) \prod_{l=1}^{\tilde{L}} p\left(\delta_{l} ; \chi\right) p\left(A_{l} \mid d_{0} ; \chi\right) p\left(\phi_{l}\right) .
\end{aligned}
$$

Expanding the received signal $\mathbf{r}$ according to (6), the expectation of SCF over link $\mathbb{e}$ becomes

$$
\begin{aligned}
& \mathbf{E}_{\mathbf{x}}\left[\left\|\mathbf{r}^{H} \mathbf{s}\left(\left\|\mathbf{p}_{u}-\mathbf{p}_{v}\right\| / c_{0}\right)\right\|^{2}\right] \\
= & \sigma^{2}\|\mathbf{s}\|^{2}+\mathbf{E}_{d_{0}}\left[\bar{A}_{0} \mathbf{E}_{\theta}\left[\left\|\mathbf{s}\left(\tau_{0}\right)^{H} \mathbf{s}\left(\left\|\mathbf{p}_{u}-\mathbf{p}_{v}\right\| / c_{0}\right)\right\|^{2}\right]\right] \\
+ & \mathbf{E}_{d_{0}}\left[\bar{L} \bar{A}_{l} \mathbf{E}_{\delta_{l}, b \mid d_{0}}\left[\mathbf{E}_{\theta}\left[\left\|\mathbf{s}\left(\tau_{l}\right)^{H} \mathbf{s}\left(\left\|\mathbf{p}_{u}-\mathbf{p}_{v}\right\| / c_{0}\right)\right\|^{2}\right]\right]\right],
\end{aligned}
$$

with the expected path power $\bar{A}_{l}=\mathbf{E}_{A_{l} \mid d_{0}}\left[A_{l}\right]$ and the expected MPC number $\bar{L}=\mathbf{E}_{L \mid d_{0}}[L]$, given the LOS distance $d_{0}$. The derivation of (35) is detailed in Appendix II. We use the notation $\tau=\left\|\mathbf{p}_{u}-\mathbf{p}_{v}\right\| / c_{0}$ in derivations for simplicity, keeping in mind that $\tau$ is a function of positions $\mathbf{p}_{u}$ and $\mathbf{p}_{v}$. Let us further assume that the symbol on each subcarrier has a constant power, for example phase-shift keying (PSK) modulated, i.e. $\left\|S_{n}\right\|=\|S\|, \forall n=-\frac{N-1}{2}, \cdots, \frac{N-1}{2}$. The $l^{\text {th }}$ 
path's cross-correlation can be further written as

$$
\begin{aligned}
& \mathbf{s}\left(\tau_{l}\right)^{H} \mathbf{s}\left(\left\|\mathbf{p}_{u}-\mathbf{p}_{v}\right\| / c_{0}\right) \\
= & \frac{1}{N} \sum_{i=1}^{N} \sum_{m, n=-\frac{N-1}{2}}^{\frac{N-1}{2}} S_{n}^{*} e^{-j n \omega\left(i T-\tau_{l}\right)} S_{m} e^{j m \omega(i T-\tau)} \\
= & \frac{1}{N} \sum_{m, n=-\frac{N-1}{2}}^{\sum_{n}^{N}} S_{m}^{*} e^{j n \omega \tau_{l}-j m \omega \tau} \underbrace{\sum_{i=1}^{N} e^{j(m-n) \omega i T}}_{=N \delta(m-n)} \\
= & \|S\|^{2} \sum_{n=-\frac{N-1}{2}}^{\frac{N-1}{2}} e^{j n \omega\left(\tau_{l}-\tau\right)}=\|S\|^{2} \frac{\sin \left(\omega N\left(\tau_{l}-\tau\right) / 2\right)}{\sin \left(\omega\left(\tau_{l}-\tau\right) / 2\right)} \\
\triangleq & \|S\|^{2} D_{l}\left(\left\|\mathbf{p}_{u}-\mathbf{p}_{v}\right\|\right),
\end{aligned}
$$

where $D_{l}\left(\left\|\mathbf{p}_{u}-\mathbf{p}_{v}\right\|\right)$ is a circular symmetric function of $\mathbf{p}_{u}$ around a given $\mathbf{p}_{v}$, obtained by rotating the order $(N-1)$ Dirichlet kernel, also known as the periodic sinc function, around $\mathbf{p}_{v}$.

\section{A. Asymptotic Localization Unbiasedness}

A condition of the DiPNet being asymptotically unbiased for location estimate is that the true position $\mathbf{p}_{u, 0}$ is a local maximum of $\log b_{u}^{(1)}$ for $\left|\mathcal{V}_{u}\right| \rightarrow \infty$. Since $D_{l}^{2}\left(\left\|\mathbf{p}_{u}-\mathbf{p}_{v}\right\|\right)$ is a smooth function for arbitrary $\left\|\mathbf{p}_{u}-\mathbf{p}_{v}\right\| \neq 0$, the asymptotic unbiasedness condition can be proven by the derivative test w.r.t. $\Delta \tau$ for $\Delta \tau \rightarrow 0$ as

$$
\text { stationarity: } \lim _{\Delta \tau \rightarrow 0} \frac{\partial \mathbf{E}_{\mathbf{x}}\left[\left\|\mathbf{r}^{H} \mathbf{s}\left(\left\|\mathbf{p}_{u}-\mathbf{p}_{v}\right\| / c_{0}\right)\right\|^{2}\right]}{\partial \Delta \tau}=0 \text {, }
$$

$$
\text { concavity: } \lim _{\Delta \tau \rightarrow 0} \frac{\partial^{2} \mathbf{E}_{\mathbf{x}}\left[\left\|\mathbf{r}^{H} \mathbf{s}\left(\left\|\mathbf{p}_{u}-\mathbf{p}_{v}\right\| / c_{0}\right)\right\|^{2}\right]}{\partial \Delta \tau^{2}}<0 \text {. }
$$

The updated logarithmic belief $\log b_{u}^{(1)}$ is asymptotically proportional to the superposition of the expected contributions from each path, over $d_{0}, \theta, b$ and $\delta_{l}$, as indicated in (35) and (36). Therefore, we evaluate the derivatives of the expected $D_{l}\left(\left\|\mathbf{p}_{u}-\mathbf{p}_{v}\right\|\right)^{2}$ of the LOS path $(l=0)$ and the MPCs $(l>0)$, w.r.t. $\Delta \tau$ for $\Delta \tau=0$. The first derivative of the expectation over $\theta$ is written as

$$
\begin{aligned}
& \lim _{\Delta \tau \rightarrow 0} \frac{\partial \mathbf{E}_{\theta}\left[D_{l}\left(\left\|\mathbf{p}_{u}-\mathbf{p}_{v}\right\|\right)^{2}\right]}{\partial \Delta \tau} \\
= & \mathbf{E}_{\theta}\left[\lim _{\Delta \tau \rightarrow 0} \frac{\partial D_{l}\left(\left\|\mathbf{p}_{u}-\mathbf{p}_{v}\right\|\right)^{2}}{\partial \Delta \tau}\right] \\
= & \mathbf{E}_{\theta}\left[2 \cos (\theta-\tilde{\theta}) \sum_{m, n=-\frac{N-1}{2}}^{\frac{N-1}{2}} j m \omega e^{j S_{m n} \omega\left(\delta_{l}+b\right)}\right]=0,
\end{aligned}
$$

with the notation $S_{m n}=m+n$, and proves the stationarity condition (37). The second derivative of the expectation over $\theta$ is expressed as

$$
\begin{aligned}
& \ddot{D}_{l}^{2}\left(\left\|\mathbf{p}_{u, 0}-\mathbf{p}_{v}\right\|\right) \\
\triangleq & \lim _{\Delta \tau \rightarrow 0} \frac{\partial^{2} \mathbf{E}_{\theta}\left[D_{l}\left(\left\|\mathbf{p}_{u}-\mathbf{p}_{v}\right\|\right)^{2}\right]}{\partial \Delta \tau^{2}} \\
= & \mathbf{E}_{\theta}\left[\lim _{\Delta \tau \rightarrow 0} \frac{\partial^{2} D_{l}\left(\left\|\mathbf{p}_{u}-\mathbf{p}_{v}\right\|\right)^{2}}{\partial \Delta \tau^{2}}\right] \\
= & -\pi \sum_{n, m=-\frac{N-1}{2}}^{\frac{N-1}{2}}\left(S_{m n}^{2} \omega^{2}+\frac{S_{m n}}{\tau_{0}} j \omega\right) e^{j S_{m n} \omega\left(\delta_{l}+b\right)} .
\end{aligned}
$$

It can be observed that the expectation of the second derivative is independent of $\tilde{\theta}$. Hence the expectation of $D_{l}\left(\left\|\mathbf{p}_{u}-\mathbf{p}_{v}\right\|\right)^{2}$ is isotropic in the sense of concavity for $\lim _{\mathbf{p}_{u} \rightarrow \mathbf{p}_{u, 0}}$. For the LOS path, inserting $\delta_{0}=0$ and $b=0$ into (40), the second order derivative states

$$
\ddot{D}_{0}^{2}\left(\tau_{0}\right)=-\frac{\pi \omega^{2} N^{2}\left(N^{2}-1\right)}{6} .
$$

The condition of concavity can be reformulated by combining (35), (36) and (38) as

$$
\varsigma \triangleq \mathbf{E}_{d_{0}}[\underbrace{\frac{6 \bar{L} \bar{A}_{l} \mathbf{E}_{\delta_{l}, b \mid d_{0}}\left[\ddot{D}_{l}^{2}\left(\left\|\mathbf{p}_{u, 0}-\mathbf{p}_{v}\right\|\right)\right]}{\pi \omega^{2} N^{2}\left(N^{2}-1\right)}-\bar{A}_{0}}_{\triangleq \tilde{\varsigma}\left(d_{0}\right)}]<0,
$$

where $\varsigma$ is dubbed the concavity indicator and $\tilde{\varsigma}\left(d_{0}\right)$ is the conditional concavity indicator (CCI) with a given $d_{0}$. The concavity condition holds, if and only if the concavity indicator $\varsigma$ is negative. Most of the communication-related channel parameters, e.g. power-delay profile, delay spread, shadow fading, LOS probability and K-factor, are intensively investigated. In comparison, the localization-related channel characteristics, for example the distribution of $\delta_{l}$ and $b$, is not always available from the study of channel model. However, for the $l^{\text {th }}$ MPC with arbitrarily distributed $\delta_{l}$ and $b$, an upper-bound of $\ddot{D}_{l}^{2}\left(\left\|\mathbf{p}_{u, 0}-\mathbf{p}_{v}\right\|\right)$ can be formulated from (40) as

$$
\begin{aligned}
\ddot{D}_{l}^{2}\left(\left\|\mathbf{p}_{u, 0}-\mathbf{p}_{v}\right\|\right) & \leq \pi \sum_{n, m=-\frac{N-1}{2}}^{\frac{N-1}{2}} S_{m n}^{2} \omega^{2}+\frac{\left\|S_{m n}\right\|}{\tau_{0}} \omega \\
& =\frac{\pi \omega^{2} N^{2}\left(N^{2}-1\right)}{6}+\frac{\pi \omega N\left(N^{2}-1\right)}{3 \tau_{0}} .
\end{aligned}
$$

With (41), (43) and $\omega N=2 \pi B$, the CCI can be upperbounded, which yields a new sufficient negative condition as

$$
\varsigma<\mathbf{E}_{d_{0}}[\underbrace{\left(1+\frac{c_{0}}{\pi B d_{0}}\right) \bar{L} \bar{A}_{l}-\bar{A}_{0}}_{\geq \tilde{\varsigma}\left(d_{0}\right)}]<0 .
$$

For a given non-zero $d_{0}$, if the expected LOS path power is larger than the expected total power of all other paths, there exists a minimal bandwidth, inversely proportional to $d_{0}$, to guarantee the concavity condition (38) holds for arbitrarily distributed $\delta_{l}$ and $b$. In a few channel models, both $\delta_{l}$ and $b$ are assumed exponentially distributed [30], 
i.e. $p(b ; X=\mathrm{NLOS})=\operatorname{Exp}\left(a_{\mathbb{B}}\right), p\left(\delta_{l} ; X=\mathrm{LOS}\right)=\operatorname{Exp}\left(a_{\mathbb{L}}\right)$ and $p\left(\delta_{l} ; X=\mathrm{NLOS}\right)=\operatorname{Exp}\left(a_{\mathbb{N}}\right)$. The rate parameters $a_{\mathbb{B}}, a_{\mathbb{L}}$ and $a_{\mathbb{N}}$ can be derived from the mean NLOS delay and delay spreads from channel models. The CCI in (42) can be expressed in close form by marginalizing over $\delta_{l}$ and $b$, as detailed in Appendix III. The CCI can be utilized to analytically assess the applicability of DiPNet, given limited system and channel characteristics. The DiPNet is an asymptotically unbiased localization algorithm if $\tilde{\varsigma}\left(d_{0}\right)<$ $0, \forall d_{0} \in\left(d_{\min }, d_{\max }\right)$, where $d_{\min }$ and $d_{\max }$ are the minimum and maximum operational distances of an application. The CCIs in typical urban and rural areas are demonstrated in Fig. 2 in Section V.

\section{B. Resistance to Erroneous Distance Information}

It is known that two-step localization approaches with Gaussian ranging model are vulnerable to large distance estimation offsets, for example due to wrongly detected paths or clock offset. In order to evaluate the erroneous distance information resistance of DiPNet, we investigate a specific scenario as follows. An agent $\mathbb{a}_{u}$ has a position belief generically modeled by a smooth isotropic unimodal pdf $b_{u}^{(0)}$, with the single mode at the origin. The logarithm belief is defined as $g\left(\mathbf{p}_{u}\right)$, which is a monotonically decreasing function of $d_{u}=\left\|\mathbf{p}_{u}\right\|$. A neighbor $\mathbb{a}_{v}$ located on the negative $x$-axis with coordinates $\mathbf{p}_{v}=\left[-d_{0}, 0\right]^{T}$, provides inter-agent distance information with a continuous measurement function $z\left(\mathbf{p}_{u}\right)$, which is a function of $d_{u v}$ and smooth at every point except $\mathbf{p}_{v}$. The new belief $h$ in logarithm domain is expressed as

$$
h\left(\mathbf{p}_{u}\right) \triangleq \log b_{u}^{(1)}\left(\mathbf{p}_{u}\right)=g\left(\mathbf{p}_{u}\right)+z\left(\mathbf{p}_{u}\right) .
$$

Let us assume $z\left(\mathbf{p}_{u}\right)$ reaches its global maximum with an additional distance offset $\delta>-d_{0}$. This offset may introduce a local maxima shift to the belief $h\left(\mathbf{p}_{u}\right)$, which is under investigation.

Lemma 1. A point $\mathbf{p}_{o x}=\left[x_{o x}, y_{o x}\right]^{T}$ is a local maximum point of $h\left(\mathbf{p}_{u}\right)$, if and only if (a) $y_{o x}=0,(b)$ it is a local maximum point over $x$-domain and (c) $x_{o x}>-d_{0}$.

Proof. See Appendix IV.

Lemma 1 indicates that it is sufficient to investigate the local maxima shift of the belief over $x$-domain only, i.e., in the direction of link $\mathbb{e}_{u v}$. We can redefine function $h, z$ and $g$ as one dimensional function of $x_{u}$ by setting $y_{u}=0$.

Theorem 1. For DiPNet, if the neighbor $\mathbb{a}_{v}$ is separated from $\mathbb{a}_{u}$ by at least a fractional of sample in distance $\varrho_{\kappa}$ defined in Appendix $V$, a path with an offset $\delta$ shifts the maximum of $\mathbb{a}_{u}$ 's position belief from the origin to a local maximum point $\mathbf{p}_{o x}=\left[x_{o x}, 0\right]^{T}$. The belief shift $\left\|x_{o x}\right\|$ is upper bounded by $\varrho_{\kappa}$ which decreases from $c_{0} / B$ to $c_{0} / 2 B$ with increasing $\|\delta\|$.

Proof. See Appendix V.

Theorem 2. In addition to Theorem 1, if the original position belief is modeled with isotropic bivariate normal distribution $b_{u}^{(0)}=\mathcal{N}\left(\mathbf{0}, \sigma_{0}^{2} \mathbf{I}\right)$, the belief shift $\left\|x_{\text {ox }}\right\|$ is more tightly bounded by

$$
\left\|x_{o x}\right\|< \begin{cases}\frac{\|\delta\|}{1+v}, & \forall\|\delta\|<\frac{c_{0}}{B} \\ \frac{\varrho_{\kappa}}{1+\frac{v}{3 \rho^{2}\left(1+\rho^{2}\right)}}, & \forall\|\delta\| \geqslant \frac{c_{0}}{B}\end{cases}
$$

where $\rho$ is inversely proportional to $B\|\delta\|$ and $\nu$ is the ratio between the the measurement and a priori belief uncertainties. Both $\rho$ and $\nu$ are defined in Appendix VI.

\section{Proof. See Appendix VI.}

The upper bound expressed in (46a) is the belief shift introduced by a two step approach, having a Gaussian ranging model with a mean biased by arbitrary $\delta$ and a variance modeled by the ranging $\mathrm{CRB}$, denoted as $\mathrm{CRB}_{l}$. With Theorem 2 we can observe that for a small distance offset, the belief shift from DiPNet is upper bounded by the shift from the two step approach, i.e. increasing with the distance offset $\|\delta\|$ and bandwidth $B$. For a large distance offset, the belief shift from DiPNet decreases with increasing $\|\delta\|$ and $B$, which is contrary to the two step approach and makes the DiPNet more resistant to erroneous distance information. The belief shift is demonstrated in Fig. 3, which is explained in more detail in Section V.

\section{REsults AND Discussion}

\section{A. Simulation Results}

We conduct simulations using an OFDM system designed for multi-link ranging [50], [51], with parameters as follows: bandwidth $B=37 \mathrm{MHz}$, number of subcarriers $N=2569$, subcarrier spacing $f_{s c}=14.65 \mathrm{KHz}$, carrier frequency $f_{c}=5.2 \mathrm{GHz}$, transmit power $P_{T x}=1 \mathrm{~mW}(0 \mathrm{dBm})$, temperature of $300 \mathrm{~K}$ for thermal noise calculation and an additional noise figure of $15 \mathrm{~dB}$.

In Fig. 2, we illustrate the CCI, $\tilde{\varsigma}\left(d_{0}\right)$ defined in Section IV-A, with the channel parameters of urban (C2) and rural area (D1) scenarios from the WINNER-II channel model [59]. For arbitrary multipath bias $\delta_{l}$ and NLOS bias $b$, the upper bound of $\tilde{\varsigma}\left(d_{0}\right)$ defined in (44) is calculated with the $K$-factor, path-loss models and LOS probability $P($ LOS $)$ listed in the WINNER-II channel model. For exponentially distributed $\delta_{l}$, delay spreads from the WINNERII channel model are additionally included to marginalize $\delta_{l}$. The distribution of NLOS bias $b$ is not included in the WINNER-II model. We assume an exponentially distributed NLOS bias with a mean of $0.3 \mu \mathrm{s}$, which is acquired by an urban area raytracing tool developed in project GREAT [60]. AWGN cases are included as benchmarks, where $\tilde{\varsigma}\left(d_{0}\right)=-\operatorname{Pr}(X=$ LOS $)$, representing the unrealistic optimal cases of perfect multipath and NLOS mitigation. For arbitrary $\delta_{l}$ and $b$, if $d_{0}>2 \mathrm{~m}$ in urban areas or $d_{0}>1 \mathrm{~m}$ in rural areas, $\tilde{\varsigma}\left(d_{0}\right)$ is negative, i.e. DiPNet is an asymptotically unbiased location estimator. For exponentially distributed $\delta_{l}$ and $b$, DiPNet is asymptotically unbiased for any $d_{0}>0.1 \mathrm{~m}$. All $\tilde{\varsigma}\left(d_{0}\right)$ are converging to the benchmarks with increasing $d_{0}$, where LOS probability becomes the decisive factor. The CCI of urban area is 


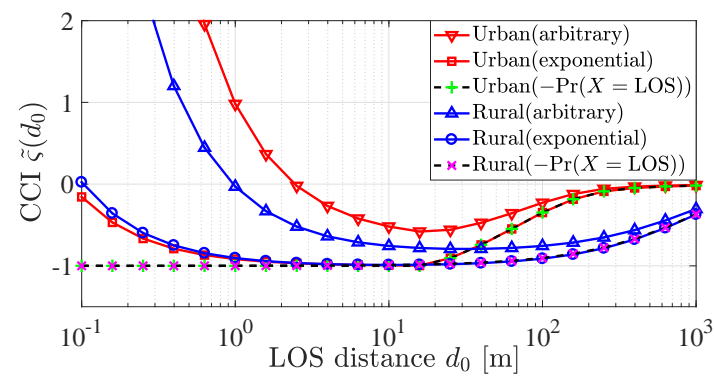

Figure 2: CCIs for urban and rural areas normalized to the LOS path power, benchmarked against the AWGN case, where $\tilde{\varsigma}\left(d_{0}\right)=-\operatorname{Pr}(X=\mathrm{LOS})$.

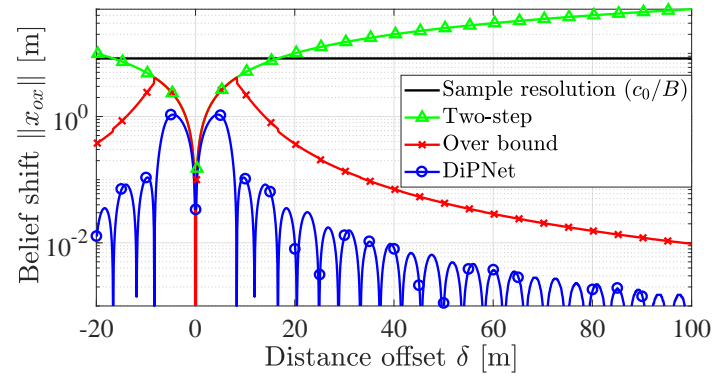

Figure 3: Belief shift of $\mathbb{a}_{u}$ 's location.

significantly larger than the one of rural area for a large $d_{0}$, due to a faster decreasing LOS probability. The investigation of the concavity indicator allows us to analytically conclude that DiPNet is asymptotically unbiased in both urban and rural areas. Besides, DiPNet in rural area may outperform the one in urban area due to a higher LOS probability.

In Fig. 3, we demonstrate Theorem 2 in Section IV-B with $\nu=1$. The location belief shift $\left\|x_{o x}\right\|$ with DiPNet (in blue), its upper bound (in red), the belief shift with the corresponding Gaussian two-step approach (in green) and a sample expressed in meters (in black) against increasing distance offset $\delta$ are plotted. The upper bound derived in Theorem 2 is always smaller than one sample distance, firstly increases then decreases as the envelope of the DiPNet belief shift and becomes negligible for large $\|\delta\|$. Whereas the belief shift of the Gaussian two-step approach monotonically increases with $\|\delta\|$. This observation verifies the erroneous distance information resistance of DiPNet.

Finally, we conduct simulations of anchor-free network localization in urban and rural area with complete channel models adapted from WINNER-II. Different sizes of fully meshed networks composed of three to thirty agents are simulated. Agents are uniformly deployed in a $100 \mathrm{~m} \times 100 \mathrm{~m}$ area. DiPNet is compared with three two-step algorithms, namely a correlation-based ranging approach [32], a SAGEbased approach for multipath mitigation [33] and a SAGEbased approach with only LOS links as a benchmark for perfect NLOS-rejection. All the two-step approaches apply the ERV concept [28] and the Gaussian ranging model, with the one-path ZZB as ranging variance [29], [61]. A DPF with 1000 particles is implemented at each agent for every algorithms with parametric belief exchanges. Fig. 4 shows the ranging RMSEs from correlation and SAGE, in comparison with the $\mathrm{CRB}$ and the ZZB. Ranging samples

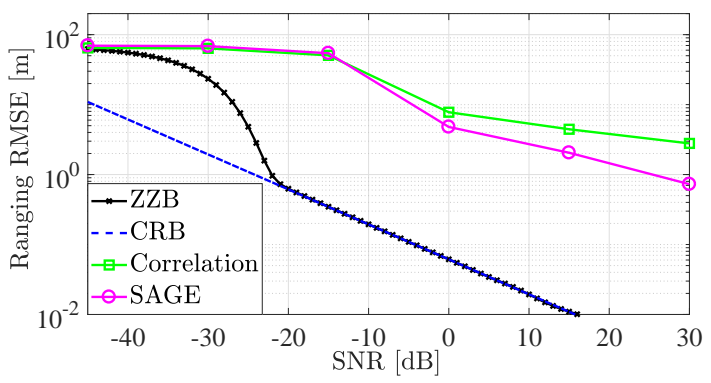

Figure 4: correlation-based and SAGE-based Ranging RMSEs in comparison with CRB and ZZB in both urban and rural areas.

are collected from all the links in the networks under investigation in both urban and rural areas. SAGE outperforms the correlation-based ranging as expected. However, both ranging RMSEs diverge from the bounds due to the unpredictable propagation effects, e.g. multipath/NLOS-bias, fading and SNR estimation error. This divergence directly limits the achievable localization accuracy for two-step approaches with the ranging error modeled by the bounds, as shown next. In Fig. 5, the anchor-free network localization performances of the compared algorithms in urban and rural areas are shown. In Fig. 5(a) and Fig. 5(b) the framework distance RMSEs defined in (4) of the compared algorithms with different network sizes are plotted. In Fig. 5(c) and Fig. 5(d) the cumulative distribution functions (CDFs) of the absolute framework distance error of different algorithms are compared for networks with three and thirty agents. The RMSEs of all algorithms decrease with an increasing number of agents from three to thirty, which indicates a cooperative gain through mesh networks. Correlation and SAGE-based algorithms result in larger RMSEs than the other two due to the NLOS-bias. The proposed DiPNet performs similarly to the NLOS-rejected SAGE, which verifies that the DiPNet is NLOS-bias resistant as proven in Section IV-B. The DiPNet obtains similar sub-meter RMSEs in both urban and rural areas for a number of agents larger than 12. The CDF plots show that in urban area, localization outliers are more often present than in rural area, except the DiPNet and NLOS-rejected SAGE in 30agent networks. It is due to the fact that the LOS probability in rural area $(95.1 \%)$ is significantly higher than the one in urban area $(65.6 \%)$ ). Both CDF and RMSE plots show a slight outperforming of DiPNet compared to the NLOSrejected SAGE in dense networks in sub-meter error range. It is caused by the non-resolvable MPCs in SAGE algorithm and approximation error in ZZB.

\section{B. Experimental Results}

We conducted two outdoor experiments with six swarm navigation prototypes developed at our research group [62], on a grass field at the German Aerospace Center (DLR). Two-way ranging between all prototypes is implemented in a sequential fashion, with OFDM signals similarly to simulations, except a transmit power of $100 \mathrm{~mW}(20 \mathrm{dBm})$ and a carrier frequency of $5.5 \mathrm{GHz}$ for forward links and $5.7 \mathrm{GHz}$ 


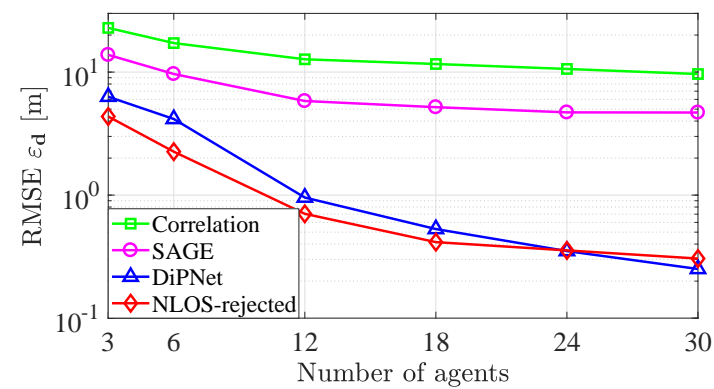

(a) Urban area RMSE $\varepsilon_{\mathbf{d}}$

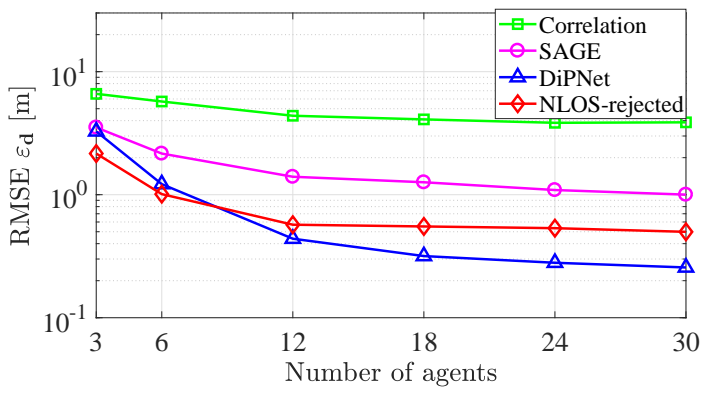

(b) Rural area RMSE $\varepsilon_{\mathbf{d}}$

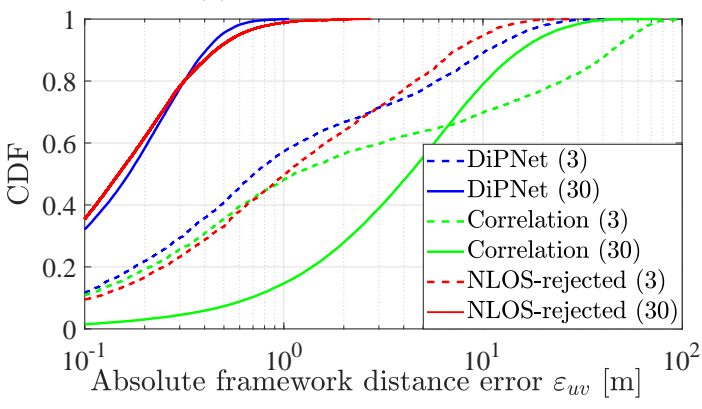

(c) Urban area CDF of $\varepsilon_{u v}$

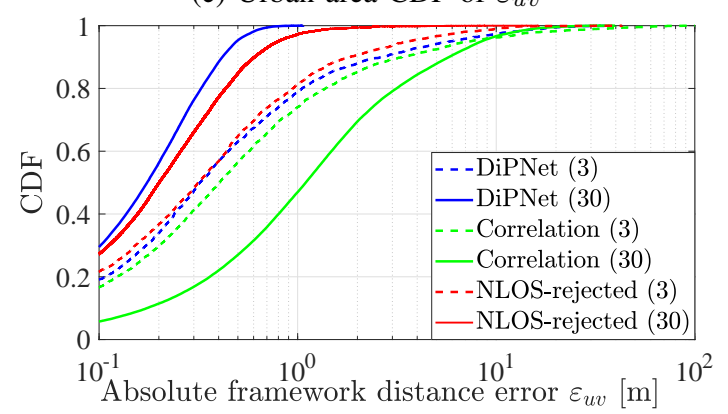

(d) Rural area CDF of $\varepsilon_{u v}$

Figure 5: Simulation results in urban and rural areas with DiPNet,correlation-based, SAGE-based and NLOS-rejected SAGE algorithms: (a) and (b) framework distance RMSE for 3 to 30 agents, (c) and (d) absolute framework distance error CDF for 3 and 30 agents.

for backward links are used. A second OFDM symbol with a scattered pilot structure is transmitted additionally for SNR estimation, counteracting non-ideal effects related to hardware. All 30 links are pre-calibrated in our laboratory over cables and radio frequency (RF) attenuators to compensate hardware characteristics like the RF front-end delays and the filter frequency responses. Five prototypes are placed in approximately symmetric pentagonal formations, with a dimension of $15 \mathrm{~m}$ in experiment 1 and a dimension of $30 \mathrm{~m}$ in experiment 2 , and remain stationary. The sixth prototype is mounted on a remotely controlled rover, driving around within a $50 \mathrm{~m} \times 80 \mathrm{~m}$ area. An accurate ground truth of agent's location is continuously obtained with a reflecting prism on the rover tracked by a tachymeter from Leica Geosystems. The received raw OFDM symbols are collected through Ethernet at a host computer and time-stamped together with the ground truth, so that the experiments can be replayed in laboratory for algorithm comparison. In total 17700 two-way measurements are collected, 260 snapshots for experiment 1 and 330 snapshots for experiment 2. Similar to simulations, we implement a SAGE-based algorithm exchanging parametric beliefs, referred to as parametric SAGE, to compare with DiPNet. In addition, sample-based DPE and SAGE approaches are implemented, where particle represented beliefs are directly exchanged and incorporated in the DPF. To maintain similar complexity for each agent, 7157 particles are used for DiPNet and parametric SAGE, whereas 100 particles are employed for sample-based DPE and sample-based SAGE. The experimental setup is shown in Fig. 6, including Fig. 6(a) images of experiments, where stationary agents are marked in yellow and rover in red, Fig. 6(b) agent's true trajectories in experiment 2 and their particle-represented beliefs from DiPNet at snapshot 61, Fig. 6(c) and Fig. 6(d) agent's true and estimated trajectories in experiment 1 from parametric SAGE and DiPNet. The rover is mostly driving smoothly with a moderate velocity, except from snapshot 250 in experiment 2, where the maximum velocity and rapid turns are experienced by the rover with the trajectory illustrated in Fig. 6(b) in red. The moving/stationary condition information is not available at agents, i.e. the DPFs at all agents apply the same mobility model. The optimal rigid affine transformation $\mathcal{T}_{\text {opt }}$ is applied to generate Fig. 6(b)-Fig. 6(d) for visualization convenience [48]. Comparing Fig. 6(c) and Fig. 6(d), we can see that DiPNet significantly outperforms parametric SAGE. A more detailed comparison can be found in Fig. 7 , with Fig. 7(a) and Fig. 7(b) showing the framework distance RMSE for each snapshot and, Fig. 7(c) and Fig. 7(d) showing the CDF of the absolute framework distance error. In both experiments, the ranging links are distorted with the MPCs from surrounding metallic structures. Additionally, low SNR is observed for some links due to the grass field ground reflection. DiPNet outperforms all three other algorithms in both experiments. Both sample-based DPE and DiPNet perform more robustly than their corresponding two-step counter partners. Sample-based DPE experiences a limited achievable accuracy due to small particle populations, as reported in [53]. A larger network dimension in experiment 2 leads to a higher failure rate for SAGEbased approaches, while only slightly affects the accuracy of DiPNet in sub-meter range. A higher rover dynamics also slightly reduces the DiPNet accuracy in sub-meter level due to a higher uncertainty in the state transition. 


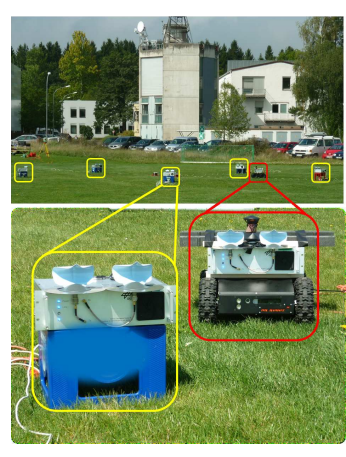

(a) Experimental setup

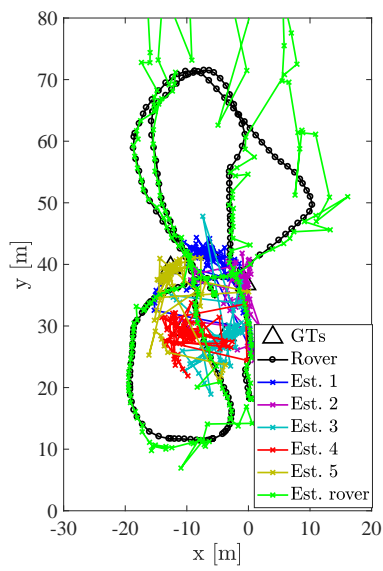

(c) parametric SAGE in Ex. 1

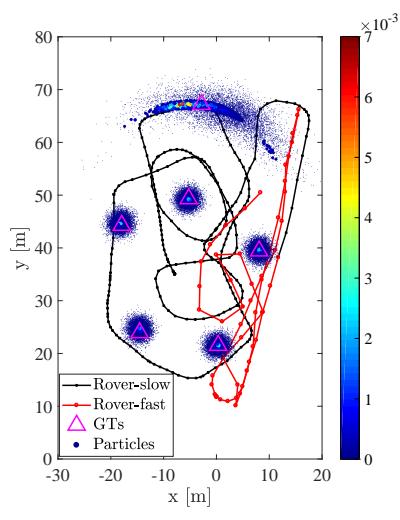

(b) DiPNet snapshot in Ex. 2

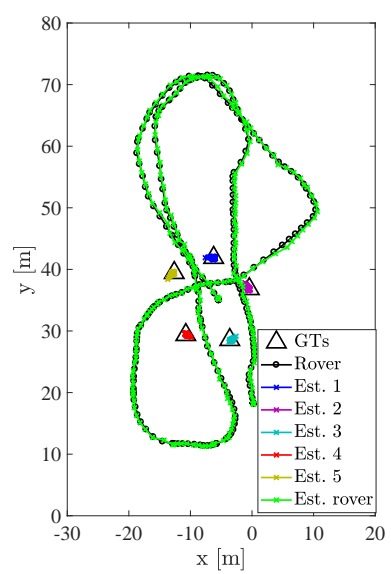

(d) DiPNet in Ex. 1
Figure 6: Experiments: (a) experimental setup, (b) agent's true trajectories in experiment 2 and particles at snapshot 61 , (c) and (d) agent's true and estimated trajectories from parametric SAGE and DiPNet in experiment 1.

\section{CONCLUSION}

We propose a DiPNet algorithm, where the location information is directly extracted from the received PHY signals. DiPNet incorporates neighbor's location uncertainty and possesses low complexity for realtime processing. Compared with the traditional two-step localization algorithms, DiPNet avoids distance estimation, which makes it more robust against unpredictable propagation effects. Through theoretical investigation, it is proven that DiPNet is an asymptotically unbiased location estimator in typical urban and rural areas, where most of the considered applications take place. DiPNet is also proven to be resistant to erroneous distance information, which is an essential advantage for multipath/NLOS-bias and outlier mitigation. Simulations with realistic WINNER-II channel models and two experiments have been conducted. DiPNet achieves a near-optimal performance and outperforms the state-of-theart algorithms, thanks to the collective PHY processing of massive links. Considering the high estimation accuracy achieved with a low complexity, DiPNet is particularly attractive for realtime localization in dense networks.

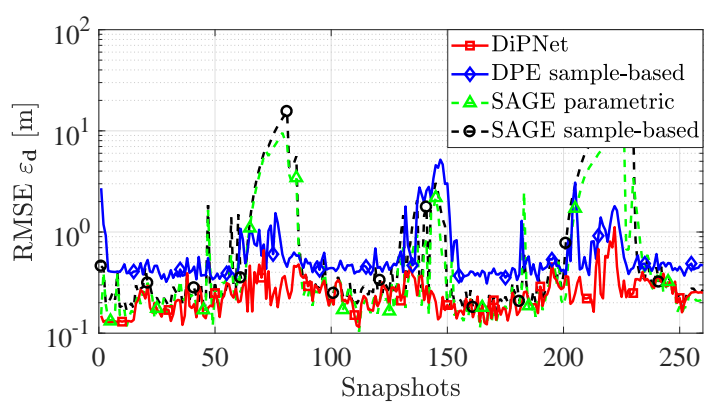

(a) Ex. 1 RMSE $\varepsilon_{\mathbf{d}}$

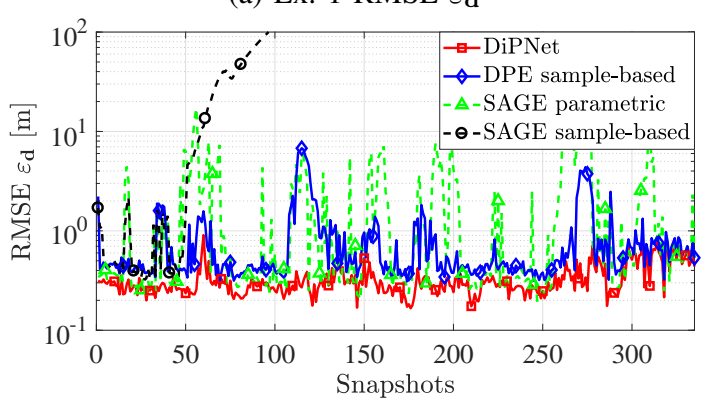

(b) Ex. 2 RMSE $\varepsilon_{\mathrm{d}}$

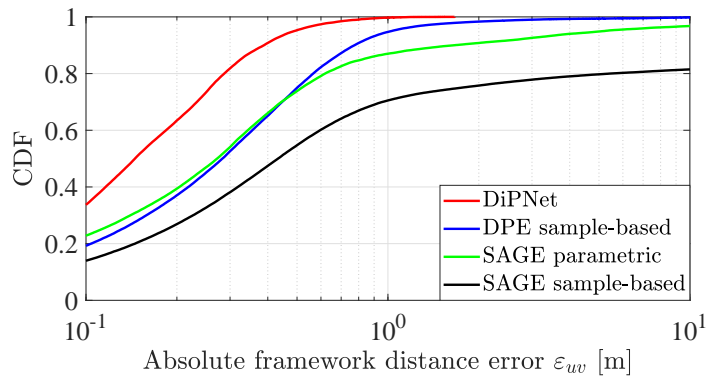

(c) Ex. $1 \mathrm{CDF}$ of $\varepsilon_{u v}$

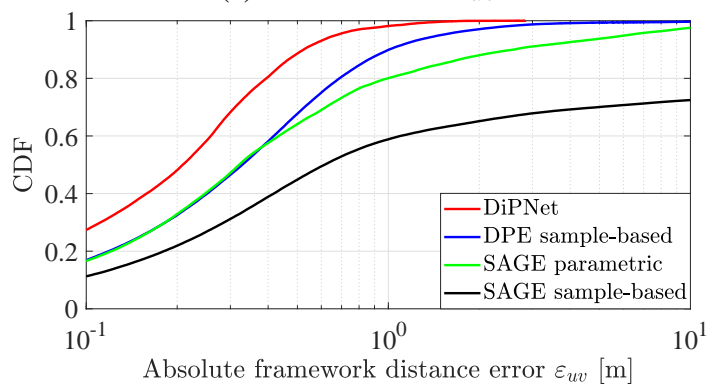

(d) Ex. $2 \mathrm{CDF}$ of $\varepsilon_{u v}$

Figure 7: Experimental performances of DiPNet, parametric SAGE, sample-based DPE and sample-based SAGE: (a) and (b) framework distance RMSE at each snapshot, (c) and (d) absolute framework distance error CDF.

\section{APPENDIX I}

DERIVATION OF EBIM IN (13)

The EBIM $\mathbf{J}_{u}$ can be formulated as

$$
\mathbf{J}_{u}=\mathbf{J}_{(u, u)}-\mathbf{D}_{u},
$$


where $\mathbf{J}_{(u, u)}$ is defined in (17). The term $\mathbf{D}_{u}$ is the information degradation due to neighbor's uncertainty

$$
\begin{aligned}
\mathbf{D}_{u} & \triangleq \sum_{v \in \mathcal{N}_{u}} \mathbf{J}_{(u, v)}^{T} \mathbf{J}_{(v, v)}^{-1} \mathbf{J}_{(u, v)}, \\
\mathbf{J}_{(u, v)} & =-\mathbf{E}_{\mathbf{p}_{u}, \mathbf{p}_{v}}\left[\nu_{u v} \mathbf{e}_{u v} \mathbf{e}_{u v}^{T}\right] \\
\mathbf{J}_{(v, v)} & =\mathbf{I}_{v}^{(k-1)}+\mathbf{E}_{\mathbf{p}_{u}, \mathbf{p}_{v}}\left[\nu_{u v} \mathbf{e}_{u v} \mathbf{e}_{u v}^{T}\right] .
\end{aligned}
$$

The superscript $(k-1)$ of $\mathbf{I}_{v}^{(k-1)}$ is omitted for simplicity. With the assumption of concentrated belief, the following approximation can be applied

$$
\mathbf{E}_{\mathbf{p}_{u}, \mathbf{p}_{v}}\left[\nu_{u v} \mathbf{e}_{u v} \mathbf{e}_{u v}^{T}\right] \approx \bar{\nu}_{u v} \overline{\mathbf{e}}_{u v} \overline{\mathbf{e}}_{u v}^{T} .
$$

Inserting I.3, (I.4) and I.5 into (I.2) and apply the ShermanMorrison formula, the information degradation can be approximated as

$$
\begin{aligned}
& \mathbf{D}_{u} \\
\approx & \sum_{v \in \mathcal{V}_{u}} \bar{\nu}_{u v}^{2} \overline{\mathbf{e}}_{u v} \overline{\mathbf{e}}_{u v}^{T}\left(\mathbf{I}_{v}^{-1}-\frac{\mathbf{I}_{v}^{-1} \bar{\nu}_{u v} \overline{\mathbf{e}}_{u v} \overline{\mathbf{e}}_{u v}^{T} \mathbf{I}_{v}^{-1}}{1+\bar{\nu}_{u v} \sigma_{v \mapsto u v}^{2}}\right) \overline{\mathbf{e}}_{u v} \overline{\mathbf{e}}_{u v}^{T} \\
= & \sum_{v \in \mathcal{V}_{u}} \bar{\nu}_{u v} \overline{\mathbf{e}}_{u v}\left(\bar{\nu}_{u v} \sigma_{v \mapsto u v}^{2}-\frac{\bar{\nu}_{u v}^{2} \sigma_{v \mapsto u v}^{4}}{1+\bar{\nu}_{u v} \sigma_{v \mapsto u v}^{2}}\right) \overline{\mathbf{e}}_{u v}^{T} .
\end{aligned}
$$

Plugging (I.6) into (I.1), the EBIM $\mathbf{J}_{u}$ is reformulated as

$$
\mathbf{J}_{u}
$$$$
\approx \mathbf{I}_{u}^{(0)}+\sum_{v \in \mathcal{V}_{u}}\left(\bar{\nu}_{u v}-\bar{\nu}_{u v}^{2} \sigma_{v \mapsto u v}^{2}+\frac{\bar{\nu}_{u v}^{3} \sigma_{v \mapsto u v}^{4}}{1+\bar{\nu}_{u v} \sigma_{v \mapsto u v}^{2}}\right) \overline{\mathbf{e}}_{u v} \overline{\mathbf{e}}_{u v}^{T} .
$$

The expression in (13) is derived by simplifying (I.7), which completes the proof.
APPENDIX II

DERIVATION OF SCF EXPECTATION IN (35)

$$
\begin{aligned}
& \mathbf{E}_{\mathbf{x}}\left[\left\|\mathbf{r}^{H} \mathbf{s}(\tau)\right\|^{2}\right] \\
= & \mathbf{E}_{\mathbf{x}}\left[\left\|\left(\sum_{l=0}^{L} \alpha_{l}^{*} \mathbf{s}\left(\tau_{l}\right)^{H}+\boldsymbol{\epsilon}^{H}\right) \mathbf{s}(\tau)\right\|^{2}\right] \\
= & \mathbf{E}_{\mathbf{x}}\left[\sum_{l=0}^{L}\left\|\alpha_{l}^{*} \mathbf{s}\left(\tau_{l}\right)^{H} \mathbf{s}(\tau)\right\|^{2}\right]+\mathbf{E}_{\mathbf{x}}\left[\left\|\boldsymbol{\epsilon}^{H} \mathbf{s}(\tau)\right\|^{2}\right] \\
+ & \underbrace{\mathbf{E}_{\mathbf{x}}\left[2 \Re\left\{\sum_{l=0}^{L} \sum_{h>l}^{L} \alpha_{l}^{*} \alpha_{h} \mathbf{s}\left(\tau_{l}\right)^{H} \mathbf{s}(\tau) \mathbf{s}(\tau)^{H} \mathbf{s}\left(\tau_{h}\right)\right\}\right]}_{(1)=0} \\
+ & \underbrace{\mathbf{E}_{\mathbf{x}}\left[2 \Re\left\{\sum_{l=0}^{L} \alpha_{l}^{*} \mathbf{s}\left(\tau_{l}\right)^{H} \mathbf{s}(\tau) \mathbf{s}(\tau)^{H} \boldsymbol{\epsilon}\right\}\right]}_{(2)=0} \\
= & \mathbf{E}_{\mathbf{x}}\left[\operatorname{Tr}\left[\sigma^{2} \mathbf{I s}(\tau) \mathbf{s}(\tau)^{H}\right]\right] \\
+ & \mathbf{E}_{d_{0}, \theta}\left[\mathbf{E}_{X \mid d_{0}}\left[\mathbf{E}_{A_{0} \mid d_{0}, X}\left[A_{0}\right]\right]\left\|\mathbf{s}\left(\tau_{0}\right)^{H} \mathbf{s}(\tau)\right\|^{2}\right] \\
+ & \mathbf{E}_{d_{0}, \theta}\left[\mathbf{E}_{X \mid d_{0}}\left[\mathbf{E}_{L, b \mid X}\left[\sum_{l=1}^{L} \mathbf{E}_{A_{l}, \delta_{l} \mid d_{0}, X}\left[A_{l}\left\|\mathbf{s}\left(\tau_{l}\right)^{H} \mathbf{s}(\tau)\right\|^{2}\right]\right]\right] .\right.
\end{aligned} .
$$

We have used the fact that paths have independent channel gains $\alpha_{l}$ with uniformly distributed phases to prove the cross-terms (1) and (2) equal to zero. The sum of expectations over all MPCs in (II.2) can be replaced with the expectation over a single MPC, since the parameters of MPCs, i.e. $A_{l}$ and $\delta_{l}$, are i.i.d., i.e.

$$
\begin{aligned}
& \mathbf{E}_{\mathbf{x}}\left[\left\|\mathbf{r}^{H} \mathbf{s}(\tau)\right\|^{2}\right] \\
= & \sigma^{2}\|\mathbf{s}\|^{2}+\mathbf{E}_{d_{0}}[\underbrace{\mathbf{E}_{A_{0} \mid d_{0}}\left[A_{0}\right]}_{\overline{A_{0}}} \mathbf{E}_{\theta}\left[\left\|\mathbf{s}\left(\tau_{0}\right)^{H} \mathbf{s}(\tau)\right\|^{2}\right]] \\
+ & \mathbf{E}_{d_{0}}[\underbrace{\mathbf{E}_{L \mid d_{0}}[L]}_{\bar{L}} \underbrace{\mathbf{E}_{A_{l} \mid d_{0}}\left[A_{l}\right]}_{\bar{A}_{l}} \mathbf{E}_{\delta_{l}, b \mid d_{0}}\left[\mathbf{E}_{\theta}\left[\left\|\mathbf{s}\left(\tau_{l}\right)^{H} \mathbf{s}(\tau)\right\|^{2}\right]\right]] .
\end{aligned}
$$

\section{APPENDIX III}

EXPECTATION OF $\ddot{D}_{l}^{2}\left(\left\|\mathbf{p}_{u, 0}-\mathbf{p}_{v}\right\|\right)$ OVER EXPONENTIALly Distributed $\delta_{l}$ AND $b$

For an MPC in NLOS case

$$
\begin{aligned}
& \mathbf{E}_{\delta_{l}, b ; X=\mathrm{NLOS}}\left[\ddot{D}_{l}^{2}\left(\left\|\mathbf{p}_{u, 0}-\mathbf{p}_{v}\right\|\right)\right] \\
= & \int_{0}^{\infty} \int_{0}^{\infty} a_{\mathbb{N}} a_{\mathbb{B}} \ddot{D}_{l}^{2}\left(\left\|\mathbf{p}_{u, 0}-\mathbf{p}_{v}\right\|\right) e^{-a_{\mathbb{N}} \delta_{l}} e^{-a_{\mathbb{B}} b} \mathrm{~d} \delta_{l} \mathrm{~d} b \\
= & \pi a_{\mathbb{N}} a_{\mathbb{B}} \\
\times & \sum_{n, m=-\frac{N-1}{2}}^{\frac{N-1}{2}} \frac{\omega^{2} S_{m n}^{2}\left(\tau_{0} \omega^{2} S_{m n}^{2}-a_{\mathbb{N}} a_{\mathbb{B}} \tau_{0}+a_{\mathbb{B}}+a_{\mathbb{N}}\right)}{\tau_{0}\left(\omega^{2} S_{m n}^{2}+a_{\mathbb{N}}^{2}\right)\left(\omega^{2} S_{m n}^{2}+a_{\mathbb{B}}{ }^{2}\right)} .
\end{aligned}
$$


For an MPC in LOS case, we can replace $a_{\mathbb{N}}$ by $a_{\mathbb{L}}$ and set $a_{\mathbb{B}} \rightarrow \infty$

$$
\begin{aligned}
& \mathbf{E}_{\delta_{l}, b ; X=\operatorname{LOS}}\left[\ddot{D}_{l}^{2}\left(\left\|\mathbf{p}_{u, 0}-\mathbf{p}_{v}\right\|\right)\right] \\
= & \pi\left(\frac{a_{\mathbb{L}}}{\tau_{0}}-a_{\mathbb{L}}^{2}\right) \sum_{n, m=-\frac{N-1}{2}}^{\frac{N-1}{2}} \frac{S_{m n}^{2} \omega^{2}}{S_{m n}^{2} \omega^{2}+a_{\mathbb{L}}^{2}} .
\end{aligned}
$$

\section{APPENDIX IV \\ PROOF OF LEMMA 1}

We first prove that local maxima of $h\left(\mathbf{p}_{u}\right)$ can only exist on the $x$-axis, by its contradiction. Assume there exists a local maximum at $\mathbf{p}_{o}=\left[x_{o}, y_{o}\right]^{T}$, where $y_{o} \neq 0$. The partial derivatives $h_{x}$ and $h_{y}$ of $h$, w.r.t. $x_{u}$ and $y_{u}$ can be expressed as

$$
\begin{aligned}
h_{x} & =\frac{\partial g}{\partial d_{u}} \frac{x_{u}}{d_{u}}+\frac{\partial z}{\partial d_{u v}} \frac{x_{u}+d_{0}}{d_{u v}}=0 \\
h_{y} & =\frac{\partial g}{\partial d_{u}} \frac{y_{u}}{d_{u}}+\frac{\partial z}{\partial d_{u v}} \frac{y_{u}}{d_{u v}}=0 .
\end{aligned}
$$

Since $y_{o} \neq 0$, from (IV.2) we have

$$
\frac{\partial z}{\partial d_{u v}} \frac{1}{d_{u v}}=-\frac{\partial g}{\partial d_{u}} \frac{1}{d_{u}} .
$$

Additionally we have $\partial g / \partial d_{u}<0$, since $g\left(\mathbf{p}_{u}\right)$ is unimodal. Inserting (IV.3) into (IV.1), we get

$$
h_{x}=-\frac{d_{0}}{d_{u}} \frac{\partial g}{\partial d_{u}}>0 .
$$

Hence $\mathbf{p}_{o}$ is not a stationary point of $h\left(\mathbf{p}_{u}\right)$, which contradicts to the assumption.

Then we apply the second derivative test with the following derivatives evaluated at $\mathbf{p}_{o x}=\left[x_{o x}, 0\right]^{T}$

$$
\begin{aligned}
& \frac{\partial d_{u v}}{\partial x_{u}}=\frac{x_{u}+d_{0}}{d_{u v}}, \frac{\partial d_{u}}{\partial x_{u}}=\frac{x_{u}}{d_{u}}, \frac{\partial d_{u v}}{\partial y_{u}}=\frac{\partial d_{u}}{\partial y_{u}}=0, \\
& \frac{\partial^{2} d_{u v}}{\partial y_{u}^{2}}=\frac{1}{d_{u v}}, \quad \frac{\partial^{2} d_{u}}{\partial y_{u}^{2}}=\frac{1}{d_{u}}, \\
& \frac{\partial^{2} d_{u v}}{\partial x_{u}^{2}}=\frac{\partial^{2} d_{u}}{\partial x_{u}^{2}}=\frac{\partial^{2} d_{u v}}{\partial x_{u} \partial y_{u}}=\frac{\partial^{2} d_{u}}{\partial x_{u} \partial y_{u}}=0, \\
& h_{x y}=0, \quad h_{x x}=\frac{\partial^{2} g}{\partial d_{u}^{2}}+\frac{\partial^{2} z}{\partial d_{u v}^{2}}, \quad h_{y y}=\frac{\partial g}{d_{u} \partial d_{u}}+\frac{\partial z}{d_{u v} \partial d_{u v}} .
\end{aligned}
$$

Combining (IV.2) and the assumption that $\mathbf{p}_{o x}$ is a local maximum point over $x$-domain, we can get

$$
h_{y}=0, \quad h_{x}=0 \text { and } h_{x x}<0 .
$$

According to the second derivative test, $\mathbf{p}_{o x}$ would be a local maximum point of $h$, if and only if $h_{x x} h_{y y}-h_{x y}^{2}>0$, i.e., $h_{y y}<0$. It can be shown after some algebra, that $h_{y y}<0$ only if $x_{o x}>-d_{0}$, which completes the proof.

\section{APPENDIX V \\ PROOF OF THEOREM 1}

The measurement function $z\left(x_{u}\right)$ can be substituted by the log-likelihood function of DiPNet

$$
z\left(x_{u}\right)=\operatorname{SNR}_{l} D_{l}\left(\left(x_{u}+d_{0}\right) / c_{0}\right)^{2} / N .
$$

In the case of $\delta>0$, a natural number is defined as $\kappa=\left\lfloor B \delta / c_{0}\right\rfloor+1 \in \mathbb{Z}^{+}$, where $x_{\kappa}=\delta-\kappa c_{0} / B$ is the $\kappa^{\text {th }}$ zero point to the left of $z\left(x_{u}\right)$ 's main peak. $\lfloor\cdot\rfloor$ denotes the floor operation. We further define $\gamma_{\kappa-1}$ as the $(\kappa-1)^{\text {th }}$ maximum point to the left of $z\left(x_{u}\right)$ 's main peak, where $\gamma_{0}=\delta$. According to the property of periodic sinc function, the value of $D_{l}\left(\left(x_{u}+d_{0}\right) / c_{0}\right)^{2}$ monotonically increases from the origin to $\gamma_{\kappa-1}$, where it reaches a maximum. In the case of $\gamma_{\kappa-1} \geqslant 0$, the derivative $z_{x}\left(x_{u}\right)$ of $z$ w.r.t. $x_{u}$ satisfies

$$
z_{x}\left(\gamma_{\kappa-1}\right)=0 \text { and } z_{x}\left(x_{u}\right)>0, \quad \forall 0 \leqslant x_{u}<\gamma_{\kappa-1} .
$$

Additionally by the definition of unimodality, the derivative $g_{x}\left(x_{u}\right)$ of $g$ w.r.t. $x_{u}$ fulfills

$$
g_{x}(0)=0 \text { and } g_{x}\left(x_{u}\right)<0, \quad \forall x_{u}>0 .
$$

Combining (V.2) and (V.3), we can get for the derivative $h_{x}\left(x_{u}\right)$

$$
h_{x}(0)>0 \text { and } h_{x}\left(\gamma_{\kappa-1}\right)<0 .
$$

Therefore, there exists $x_{o x} \in\left(0, \gamma_{\kappa-1}\right]$, so that

$$
\begin{aligned}
& h_{x}\left(x_{u}\right) \geqslant 0, \forall x_{u} \in\left(0, x_{o x}\right] \\
& h_{x}\left(x_{u}\right)<0, \forall x_{u} \in\left(x_{o x}, \gamma_{\kappa-1}\right],
\end{aligned}
$$

where the equality only holds at $x_{o x}$. According to Lemma 1 , the maximum point of the belief is shifted from the origin to $\mathbf{p}_{o x}$, which introduces a bias to the belief $\left\|x_{o x}\right\|<$ $\left\|\gamma_{\kappa-1}\right\|<\varrho_{\kappa} \triangleq\left\|\gamma_{\kappa-1}-x_{\kappa}\right\|$. The second inequality is obtained by the property of periodic sinc function that $\left\|x_{\kappa-1}-\gamma_{\kappa-1}\right\|<\left\|\gamma_{\kappa-1}-x_{\kappa}\right\|$. The upper bound $\varrho_{\kappa}$ decreases with increasing $\kappa$ from $\varrho_{1}=c_{0} / B$ and quickly approaches its asymptotic value $\varrho_{\infty}=c_{0} / 2 B$. The proof can be extended to $-d_{0}<\delta<0$ and $\gamma_{\kappa-1}<0$ in a similar manner, which completes the proof of Theorem 1.

\section{APPENDIX VI PROOF OF THEOREM 2}

The measurement function $z\left(x_{u}\right)$ can be approximated by its second-order Taylor expansion $\tilde{z}\left(x_{u}\right)$ at $x_{u}=\gamma_{\kappa-1}$

$$
\begin{aligned}
\tilde{z}\left(x_{u}\right) & =\frac{1}{2} \underbrace{z_{x x}\left(\gamma_{\kappa-1}\right)}_{<0}\left(x_{u}-\gamma_{\kappa-1}\right)^{2} \\
& +\underbrace{z_{x}\left(\gamma_{\kappa-1}\right)}_{=0}\left(x_{u}-\gamma_{\kappa-1}\right)+z\left(\gamma_{\kappa-1}\right) .
\end{aligned}
$$

The maximum point $\tilde{x}_{o x}$ of $g\left(x_{u}\right)+\tilde{z}\left(x_{u}\right)$ can be calculated with the equality of their derivatives

$$
\begin{aligned}
g_{x}\left(x_{u}\right)+\tilde{z}_{x}\left(x_{u}\right) & =-\frac{1}{\sigma_{0}^{2}} x_{u}+z_{x x}\left(\gamma_{\kappa-1}\right)\left(x_{u}-\gamma_{\kappa-1}\right)=0 \\
\tilde{x}_{o x} & =\frac{\gamma_{\kappa-1}}{1-\frac{1}{\sigma_{0}^{2} z_{x x}\left(\gamma_{\kappa-1}\right)}} . \quad \text { (VI.2) }
\end{aligned}
$$

With the property of the periodic sinc function, the derivatives of $z\left(x_{u}\right)$ and its Taylor expansion fulfill

$$
0 \leqslant\left\|z_{x}\left(x_{u}\right)\right\| \leqslant\left\|\tilde{z}_{x}\left(x_{u}\right)\right\|,
$$


where both equalities hold only for $x_{u}=\gamma_{\kappa-1}$. Therefore, the bias $\left\|x_{o x}\right\|$ of position belief $h\left(\mathbf{p}_{u}\right)$ is smaller than $\left\|\tilde{x}_{o x}\right\|$. Then we derive the derivatives of $z\left(x_{u}\right)$, simplifying the periodic sinc function with the sinc function, and defining $\zeta=\omega\left(\delta-\gamma_{\kappa-1}\right) / 2 c_{0}$

$$
\begin{aligned}
z_{x}\left(\gamma_{\kappa-1}\right) & \approx \frac{\omega \mathrm{SNR}_{l} \sin ^{2}(N \zeta)}{c_{0} \zeta^{2}}\left(\frac{1}{N \zeta}-\frac{\cos (N \zeta)}{\sin (N \zeta)}\right)=0, \\
z_{x x}\left(\gamma_{\kappa-1}\right) & \approx \frac{\omega^{2} \mathrm{SNR}_{l} \sin ^{2}(N \zeta)}{c_{0}^{2} \zeta^{2}} \\
& \times\left(\frac{3}{2 N \zeta^{2}}-\frac{N}{2}-\frac{2 \cos (N \zeta)}{\zeta \sin (N \zeta)}+\frac{N \cos ^{2}(N \zeta)}{2 \sin ^{2}(N \zeta)}\right) .
\end{aligned}
$$

If $\|\delta\|<c_{0} / B, \kappa=1$, i.e., $\zeta=0$. The second derivative $z_{x x}$ reaches its global minimum

$$
\lim _{\zeta \rightarrow 0} z_{x x}\left(\gamma_{\kappa-1}\right)=-\frac{\omega^{2} N^{3} \mathrm{SNR}_{l}}{6 c_{0}^{2}}=-\frac{1}{\mathrm{CRB}_{l}}=-\frac{1}{v \sigma_{0}^{2}} .
$$

The bias of position belief is bounded by

$$
\left\|x_{o x}\right\|<\left\|\tilde{x}_{o x}\right\|=\frac{\|\delta\|}{1+\frac{3 c_{0}^{2}}{2 \pi^{2} N \mathrm{SNR}_{l} B^{2} \sigma_{0}^{2}}}=\frac{\|\delta\|}{1+v} .
$$

If $\|\delta\| \geqslant c_{0} / B$

$$
\begin{aligned}
& z_{x x}\left(\gamma_{\kappa-1}\right) \\
\approx & \frac{\omega^{2} \mathrm{SNR}_{l}}{c_{0}^{2} \zeta^{2}}\left(\frac{N \cos ^{2}(N \zeta)}{2}-\frac{N \sin ^{2}(N \zeta)}{2}-\frac{\sin ^{2}(N \zeta)}{2 N \zeta^{2}}\right) \\
= & \frac{\omega^{2} \mathrm{SNR}_{l}}{c_{0}^{2} \zeta^{2}}\left(\frac{N \cos (2 N \zeta)}{2}-\frac{1-\cos (2 N \zeta)}{4 N \zeta^{2}}\right) \\
> & -\frac{\omega^{2} \mathrm{SNR}_{l}}{2 c_{0}^{2} \zeta^{2}}\left(N+\frac{1}{N \zeta^{2}}\right) \\
= & -\frac{2 N \mathrm{SNR}_{l}}{\left(\delta-\gamma_{\kappa-1}\right)^{2}}\left(1+\frac{c_{0}^{2}}{\pi^{2} B^{2}\left(\delta-\gamma_{\kappa-1}\right)^{2}}\right) \\
> & -\frac{2 N \mathrm{SRR}_{l}}{\left(\|\delta\|-\varrho_{\kappa}\right)^{2}}\left(1+\rho^{2}\right),
\end{aligned}
$$

where $\rho=c_{0} / \pi B\left(\|\delta\|-\varrho_{\kappa}\right)$. Therefore

$$
\left\|x_{o x}\right\|<\left\|\tilde{x}_{o x}\right\|<\frac{\varrho_{\kappa}}{1+\frac{v}{3 \rho^{2}\left(1+\rho^{2}\right)}} .
$$

Combining (VI.4) and (VI.6), we have the inequality (46), which completes the proof.

\section{ACKNOWLEDGMENT}

This work was partly supported by the Helmholtz Association project ARCHES (ZT-0033) and the German Research Foundation (DFG) project ModeNAV (FI 2176/1-1 and HO 2226/17-1).

\section{REFERENCES}

[1] G. Soatti, M. Nicoli, N. Garcia, B. Denis, R. Raulefs, and H. Wymeersch, "Implicit cooperative positioning in vehicular networks," IEEE Trans. Intell. Transp. Syst., vol. 19, no. 12, pp. 3964-3980, Dec. 2018

[2] E. A. de Reyna, P. Closas, D. Dardari, and P. M. Djuric, "Crowdbased learning of spatial fields for the internet of things: From harvesting of data to inference," IEEE Signal Process. Mag., vol. 35 no. 5, pp. 130-139, Sep. 2018.
[3] R. D. Taranto, S. Muppirisetty, R. Raulefs, D. Slock, T. Svensson, and $\mathrm{H}$. Wymeersch, "Location-aware communications for $5 \mathrm{G}$ networks: How location information can improve scalability, latency, and robustness of 5G," IEEE Signal Process. Mag., vol. 31, no. 6, pp. 102-112, Nov. 2014.

[4] R. M. Buehrer, H. Wymeersch, and R. M. Vaghefi, "Collaborative sensor network localization: Algorithms and practical issues," Proc. IEEE, vol. 106, no. 6, pp. 1089-1114, Jun. 2018.

[5] M. Z. Win, F. Meyer, Z. Liu, W. Dai, S. Bartoletti, and A. Conti, "Efficient multisensor localization for the internet of things: Exploring a new class of scalable localization algorithms," IEEE Signal Process. Mag., vol. 35, no. 5, pp. 153-167, Sep. 2018.

[6] B. J. Julian, M. Angermann, M. Schwager, and D. Rus, "Distributed robotic sensor networks: An information-theoretic approach," The International Journal of Robotics Research, vol. 31, no. 10, pp. 1134 1154, Aug. 2012.

[7] N. Patwari, A. O. Hero, M. Perkins, N. S. Correal, and R. J. O'Dea, "Relative location estimation in wireless sensor networks," IEEE Trans. Signal Process., vol. 51, no. 8, pp. 2137-2148, Aug. 2003.

[8] W. F. Truszkowski, M. G. Hinchey, J. L. Rash, and C. A. Rouff, "Autonomous and autonomic systems: A paradigm for future space exploration missions," IEEE Trans. Syst., Man, Cybern. C, vol. 36, no. 3, pp. 279-291, May 2006.

[9] S. Zhang, R. Raulefs, and A. Dammann, "Location information driven formation control for swarm return-to-base application," in $201624^{\text {th }}$ European Signal Processing Conference (EUSIPCO), Aug. 2016, pp. 758-763.

[10] J. del Peral-Rosado, R. Raulefs, J. López-Salcedo, and G. SecoGranados, "Survey of cellular mobile radio localization methods: From $1 \mathrm{G}$ to 5G," IEEE Commun. Surveys Tuts., vol. 20, no. 2, pp. 1124-1148, 2018.

[11] A. Shahmansoori et al., "Position and orientation estimation through millimeter-wave MIMO in 5G systems," IEEE Trans. Wireless Commun., vol. 17, no. 3, pp. 1822-1835, Mar. 2018.

[12] Y. Liu, Y. Shen, and M. Z. Win, "Single-anchor localization and synchronization of full-duplex agents," IEEE Trans. Commun., pp. 1-1, Oct. 2018.

[13] S. Zhang, T. Jost, R. Pöhlmann, A. Dammann, D. Shutin, and P. A. Hoeher, "Spherical wave positioning based on curvature of arrival by an antenna array," IEEE Wireless Commun. Lett., pp. 1-1, Oct. 2018.

[14] H. Wymeersch, J. Lien, and M. Win, "Cooperative localization in wireless networks," Proc. IEEE, vol. 97, no. 2, pp. 427-450, Feb. 2009.

[15] Y. Shen, H. Wymeersch, and M. Win, "Fundamental limits of wideband localization, part II: Cooperative networks," IEEE Trans. Inf. Theory, vol. 56, no. 10, pp. 4981-5000, Oct. 2010.

[16] A. Dammann, R. Raulefs, and S. Zhang, "On prospects of positioning in 5G," in 2015 IEEE International Conference on Communication Workshop (ICCW), Jun. 2015, pp. 1207-1213.

[17] Y. Zhu, A. Jiang, and H. K. Kwan, "ADMM-based sensor network localization using low-rank approximation," IEEE Sensors J., vol. 18, no. 20, pp. 8463-8471, Oct. 2018.

[18] A. T. Ihler, J. W. Fisher, R. L. Moses, and A. S. Willsky, "Nonparametric belief propagation for self-localization of sensor networks," IEEE J. Sel. Areas Commun., vol. 23, no. 4, pp. 809-819, Apr. 2005.

[19] N. Patwari, J. N. Ash, S. Kyperountas, A. O. Hero, R. L. Moses, and N. S. Correal, "Locating the nodes: cooperative localization in wireless sensor networks," IEEE Signal Process. Mag., vol. 22, no. 4, pp. 54-69, Jul. 2005.

[20] G. Mao, B. Fidan, and B. D. O. Anderson, "Wireless sensor network localization techniques," Comput. Netw., vol. 51, no. 10, pp. 2529-2553, Jul. 2007. [Online]. Available: http://dx.doi.org/10.1016/j.comnet.2006.11.018

[21] P. Closas and A. Gusi-Amigo, "Direct position estimation of GNSS receivers: Analyzing main results, architectures, enhancements, and challenges," IEEE Signal Process. Mag., vol. 34, no. 5, pp. 72-84, Sep. 2017.

[22] M. Z. Win, Y. Shen, and W. Dai, "A theoretical foundation of network localization and navigation," Proc. IEEE, vol. 106, no. 7, pp. 11361165, Jul. 2018

[23] P. Biswas and Yinyu Ye, "Semidefinite programming for ad hoc wireless sensor network localization," in Third International Symposium on Information Processing in Sensor Networks, 2004. IPSN 2004, Apr. 2004, pp. 46-54.

[24] L. Liao, J. Hightower, D. Fox, G. Borriello, and D. Schulz, "Bayesian filtering for location estimation," IEEE Pervasive Comput., vol. 2, no. 03, pp. 24-33, jul. 2003. 
[25] C. Savarese, J. M. Rabaey, and K. Langendoen, "Robust positioning algorithms for distributed ad-hoc wireless sensor networks," in Proceedings of the General Track of the Annual Conference on USENIX Annual Technical Conference, ser. ATEC '02. Berkeley, CA, USA: USENIX Association, 2002, pp. 317-327. [Online]. Available: http://dl.acm.org/citation.cfm?id=647057.713854

[26] F. Meyer, O. Hlinka, and F. Hlawatsch, "Sigma point belief propagation," IEEE Signal Process. Lett., vol. 21, no. 2, pp. 145-149, Feb. 2014.

[27] O. Hlinka, F. Hlawatsch, and P. M. Djuric, "Distributed particle filtering in agent networks: A survey, classification, and comparison," IEEE Signal Process. Mag., vol. 30, no. 1, pp. 61-81, Jan. 2013.

[28] S. Zhang, R. Raulefs, A. Dammann, and S. Sand, "System-level performance analysis for Bayesian cooperative positioning: From global to local," in 2013 International Conference on Indoor Positioning and Indoor Navigation (IPIN), Oct. 2013.

[29] D. Dardari, A. Conti, U. Ferner, A. Giorgetti, and M. Z. Win, "Ranging with ultrawide bandwidth signals in multipath environments,' Proc. IEEE, vol. 97, no. 2, pp. 404-426, Feb. 2009.

[30] W. Wang, T. Jost, and A. Dammann, "Estimation and modelling of NLoS time-variant multipath for localization channel model in mobile radios," in 2010 IEEE Global Telecommunications Conference (GLOBECOM 2010), Dec. 2010, pp. 1-6.

[31] Y. Shen and M. Z. Win, "Fundamental limits of wideband localization- part I: A general framework," IEEE Trans. Inf. Theory, vol. 56, no. 10, pp. 4956-4980, Oct. 2010.

32] C. Gentner, S. Sand, and A. Dammann, "OFDM indoor positioning based on TDOAs: Performance analysis and experimental results," in 2012 IEEE International Conference on Localization and GNSS (ICL-GNSS). IEEE, Jun. 2012, pp. 1-7.

[33] J. Fessler, A. O. Hero et al., "Space-alternating generalized expectation-maximization algorithm," IEEE Trans. Signal Process. vol. 42, no. 10, pp. 2664-2677, Oct. 1994.

[34] T. Jost, W. Wang, U. Fiebig, and F. Perez-Fontan, "Detection and tracking of mobile propagation channel paths," IEEE Trans. Antennas Propag., vol. 60, no. 10, pp. 4875-4883, Oct. 2012

[35] D. Jin, F. Yin, C. Fritsche, A. M. Zoubir, and F. Gustafsson, "Efficien cooperative localization algorithm in LOS/NLOS environments," in $201523^{\text {rd }}$ European Signal Processing Conference (EUSIPCO), Aug. 2015, pp. 185-189.

[36] T. Van Nguyen, Y. Jeong, H. Shin, and M. Win, "Machine learning for wideband localization," IEEE J. Sel. Areas Commun., vol. 33 , no. 7, pp. 1357-1380, Jul. 2015.

[37] S. Maranò, W. M. Gifford, H. Wymeersch, and M. Z. Win, "NLOS identification and mitigation for localization based on UWB experimental data," IEEE J. Sel. Areas Commun., vol. 28, no. 7, pp. 1026 1035, Sep. 2010

[38] I. Guvenc and C. Chong, "A survey on TOA based wireless localization and NLOS mitigation techniques," IEEE Commun. Surveys Tuts., vol. 11, no. 3, pp. 107-124, 2009.

[39] C. Gentner et al., "Multipath assisted positioning with simultaneous localization and mapping," IEEE Trans. Wireless Commun., vol. 15 no. 9, pp. 6104-6117, Sep. 2016.

[40] R. Mendrzik, H. Wymeersch, G. Bauch, and Z. Abu-Shaban, "Harnessing NLOS components for position and orientation estimation in 5G millimeter wave MIMO," IEEE Trans. Wireless Commun., vol. 18 no. 1, pp. 93-107, Jan. 2019.

[41] K. Witrisal, P. Meissner, E. Leitinger, Y. Shen, C. Gustafson, F. Tufvesson, K. Haneda, D. Dardari, A. F. Molisch, A. Conti, and M. Z. Win, "High-accuracy localization for assisted living: 5G systems will turn multipath channels from foe to friend," IEEE Signal Process. Mag., vol. 33, no. 2, pp. 59-70, Mar. 2016.

[42] A. J. Weiss, "Direct geolocation of wideband emitters based on delay and Doppler," IEEE Trans. Signal Process., vol. 59, no. 6, pp. 25132521, Jun. 2011.

[43] P. Closas and C. Fernández-Prades, "Bayesian nonlinear filters for direct position estimation," in 2010 IEEE Aerospace Conference, Mar. 2010, pp. 1-12.

[44] O. Daniel, E. Lohan, and J. Nurmi, "Relaxed direct position estimation as strategy for open-loop GNSS receivers," in 2015 International Conference on Location and GNSS (ICL-GNSS), Jun. 2015, pp. 1-7.

[45] X. Yin, T. Pedersen, P. Blattnig, A. Jaquier, and B. Fleury, "A singlestage target tracking algorithm for multistatic DVB-T passive radar systems," in 2009 IEEE 13th Digital Signal Processing Workshop and 5th IEEE Signal Processing Education Workshop, Jan. 2009, pp. $518-523$
[46] B. Krach, Sensor Fusion by Bayesian Filtering for Seamless Pedestrian Navigation. Der Andere Verlag, 2010.

[47] S. Zhang, E. Staudinger, W. Wang, C. Gentner, A. Dammann, and E. Sandgren, "DiPLoc: Direct signal domain particle filtering for network localization," in Proceedings of the $28^{\text {th }}$ International Technical Meeting of The Satellite Division of the Institute of Navigation (ION GNSS+ 2015), Tampa, Florida, Sep. 2015, pp. 2267-2274

[48] J. N. Ash and R. L. Moses, "On the relative and absolute positioning errors in self-localization systems," IEEE Trans. Signal Process. vol. 56, no. 11, pp. 5668-5679, Nov. 2008.

[49] J. Aspnes, T. Eren, D. K. Goldenberg, A. S. Morse, W. Whiteley, Y. R. Yang, B. D. O. Anderson, and P. N. Belhumeur, "A theory of network localization,” IEEE Trans. Mobile Comput., vol. 5, no. 12, pp. 1663-1678, Dec. 2006.

[50] E. Staudinger, S. Zhang, and A. Dammann, "Cramer-Rao lower bound for round-trip delay ranging with subcarrier-interleaved OFDMA," IEEE Trans. Aerosp. Electron. Syst., vol. 52, no. 6, pp. 2961-2972, Dec. 2016.

[51] S. Zhang, S. Sand, R. Raulefs, and E. Staudinger, "Self-organized hybrid channel access method for an interleaved RTD-based swarm navigation system," in $201310^{\text {th }}$ Workshop on Positioning, Navigation and Communication (WPNC), Dresden, Germany, Mar. 2013.

[52] W. Gao, X. Chen, and J. Lu, "Cubature belief propagation for self-localization of wireless networks," in 2015 IEEE International Conference on Communications (ICC), Jun. 2015, pp. 6658-6662.

[53] J. Lien, U. J. Ferner, W. Srichavengsup, H. Wymeersch, and M. Z. Win, "A comparison of parametric and sample-based message representation in cooperative localization," International Journal of Navigation and Observation, 2012.

[54] P. Tichavsky, C. H. Muravchik, and A. Nehorai, "Posterior CramérRao bounds for discrete-time nonlinear filtering," IEEE Trans. Signal Process., vol. 46, no. 5, pp. 1386-1396, May 1998.

[55] G. Golub and V. Pereyra, "The differentiation of pseudo-inverses and nonlinear least squares problems whose variables separate," SIAM Journal on Numerical Analysis, vol. 10, no. 2, pp. 413-432, Apr. 1973.

[56] C. Gentner, S. Zhang, and T. Jost, "Log-PF: Particle filtering in logarithm domain," Journal of Electrical and Computer Engineering, vol. 2018, 2018.

[57] J. Selva, "Interpolation of bounded bandlimited signals and applications," IEEE Trans. Signal Process., vol. 54, no. 11, pp. 4244-4260, Nov. 2006.

[58] M. Lashley, D. M. Bevly, and J. Y. Hung, "Performance analysis of vector tracking algorithms for weak GPS signals in high dynamics," IEEE J. Sel. Topics Signal Process., vol. 3, no. 4, pp. 661-673, Aug 2009.

[59] "WINNER II Deliverable D1.1.2: WINNER II Channel Models," Sep. 2007. [Online]. Available: http://www.istwinner.org/deliverables.html

[60] S. Sand, Hybridization with Localization Information from Wireless Communications Systems. Springer Netherlands, 2015, pp. 169-208.

[61] S. Zhang and R. Raulefs, "Multi-agent flocking with noisy anchorfree localization," in 2014 IEEE $11^{\text {th }}$ International Symposium on Wireless Communications Systems (ISWCS). IEEE, Aug. 2014, pp. 927-933.

[62] E. Staudinger and A. Dammann, "Round-trip delay ranging with OFDM signals- performance evaluation with outdoor experiments," in $201411^{\text {th }}$ Workshop on Positioning, Navigation and Communication (WPNC), Mar. 2014, pp. 1-6.

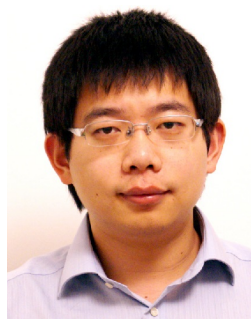

Siwei Zhang (M'14) received his M.Sc. degree in Communication Engineering in 2011 from the Technical University of Munich, Germany and his B.Sc. degree in Electrical Engineering in 2009 from Zhejiang University, China. In 2012 he joined the Institute of Communications and Navigation, German Aerospace Center, Germany as a Scientific Staff. His research interests include cooperative positioning and swarm navigation. 


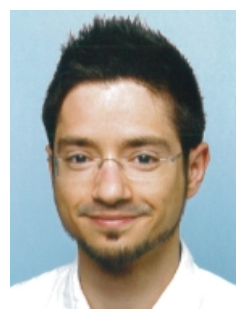

Emanuel Staudinger received the M.Sc. degree in Embedded Systems Design from the University of Applied Sciences of Hagenberg, Austria, in 2010. Since 2010, he is with the Institute of Communications and Navigation of the German Aerospace Center (DLR), Wessling, Germany. He received the $\mathrm{Ph}$.D. degree with distinction at the Institute of Electrodynamics and Microelectronics of the University of Bremen in 2015. Since then, he is leading the topic "Swarm Navigation" within the Mobile Radio Transmission Group His current research interests and activities include system design for radio based localization and time synchronization for robot swarms, experimental platform design, and validation through measurement campaigns.

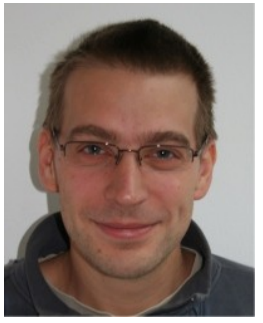

Thomas Jost (M'11) received a Diploma degree (FH) 2001 in Electrical Engineering from University of Applied Science Wiesbaden, Germany and a Diploma degree in 2003 in Electrical Engineering and Information Technology from Technical University of Darmstadt, Germany. In 2013, he received his $\mathrm{PhD}$ from University of Vigo, Spain From 2003 to 2006 he held a research assistant position in the Signal Processing Group at TU Darmstadt. From 2006 to 2018 he was a member of the scientific staff of the Institute of Communications and Navigation at the German Aerospace Center. Since 2019 he is working for Continental AG on radar systems.

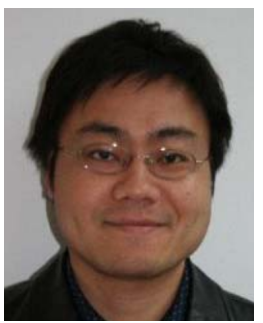

Wei Wang (M'09) received the Bachelor degree in the field of communications engineering from University of Wuhan, China, in 2003, the Master degree from University of Kiel, Germany, in 2006 and the Doctoral degree with summa cum laude from University of Erlangen-Nuremberg, Germany, in 2014.

From 2007 to 2018, he has worked as a scientific staff member at the Institute of Communications and Navigation of German Aerospace Center (DLR), Oberpfaffenhofen, Germany. Now he is a professor with the School of Information Engineering at Chang' an University, China. His research interests includes time variant parameter estimation, channel modeling, channel measurements, terrestrial radio based positioning/navigation and related topics. He received the best presentation paper award in ION GNSS 2012, best paper award in EUCAP 2018 and in COTA 2018. He is an associate editor in IET Microwave, Antennas \& Propagation, a guest editor of IEEE Access, and served as TPC member for ICC and VTC conference and workshops.

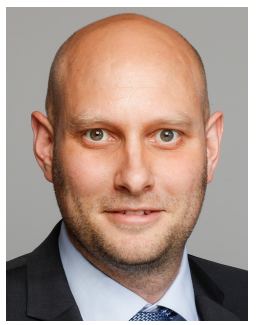

Christian Gentner studied electrical engineering at the University of Applied Science in Ravensburg, with the main topic communication technology and received his Dipl.-Ing. (BA) degree in 2006. During this study he received practical experiences at Rohde \& Schwarz in Munich. He continued his study at the University of Ulm in 2006. He received the M.Sc. and Dr.-Ing. $(\mathrm{PhD})$ degree in 2009 and 2018 respectively, both from the University of Ulm. Since 2009 , he is working at the Institute of Communications an Navigation of the German Aerospace Center (DLR). His current research focuses on indoor positioning.

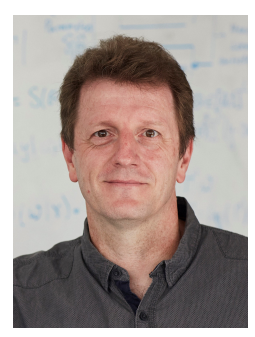

Armin Dammann (M'12) received the Dipl.-Ing. (M.Sc.) and Dr.-Ing. (Ph.D.) degrees in Electrical Engineering from the University of Ulm, Germany, in 1997 and 2005 respectively. In 1997 he joined the Institute of Communications and Navigation of the German Aerospace Center (DLR) as a research staff member. Since 2005 he is head of the Mobile Radio Transmission Research Group. His research interest and activities include signal design and signal processing for terrestrial wireless communication and navigation systems. In these fields, he has been active in several EU-projects, e.g., WINNER, WHERE and WHERE2. Armin Dammann is lecturer at the Technical University of Munich for "Robot and Swarm Navigation".

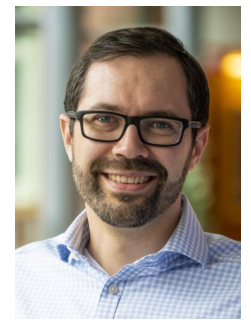

Henk Wymeersch (S'01, M'05) obtained the Ph.D. degree in Electrical Engineering/Applied Sciences in 2005 from Ghent University, Belgium. He is currently a Professor of Communication Systems with the Department of Electrical Engineering at Chalmers University of Technology, Sweden. Prior to joining Chalmers, he was a postdoctoral researcher with the Laboratory for Information and Decision Systems at the Massachusetts Institute of Technology. Prof. Wymeersch served as Associate Editor for IEEE Communication Letters, IEEE Transactions on Wireless Communications, and IEEE Transactions on Communications. His current research interests include cooperative positioning systems and intelligent transportation systems.

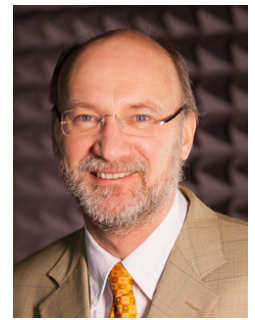

Peter A. Hoeher (F'14) received Dipl.-Ing. (M.Sc.) and Dr.-Ing. (Ph.D.) degrees in electrical engineering from the RWTH Aachen University, Aachen, Germany, and the University of Kaiserslautern, Kaiserslautern, Germany, in 1986 and 1990, respectively. From 1986 till 1998 he has been with the German Aerospace Center (DLR), Oberpfaffenhofen, Germany. Between December 1991 and November 1992 he was on leave at AT\&T Bell Laboratories, Murray Hill, NJ.

In 1998 he joined the University of Kiel, Germany, where he is a full professor of electrical and information engineering. His research interests are in the general area of communication theory and applied information theory with applications in wireless radio communications, optical wireless communications, molecular communications, underwater communications, and simultaneous wireless information and power transfer.

Since 2014 he is an IEEE Fellow "for contributions to decoding and detection that include reliability information". Dr. Hoeher received the Hugo-Denkmeier-Award '90, the ITG Award '07, and several best paper awards. Between 1999 and 2006 he served as an Associated Editor for IEEE Transactions on Communications. 